\title{
CFD-Based Structural Optimization of Rotor Cage for High-Efficiency Rotor Classifier
}

\author{
Xinliang Mou, Fangchao Jia, Ying Fang * and Chuanwen Chen *
}

check for updates

Citation: Mou, X.; Jia, F.; Fang, Y.; Chen, C. CFD-Based Structural Optimization of Rotor Cage for High-Efficiency Rotor Classifier. Processes 2021, 9, 1148. https:// doi.org/10.3390/pr9071148

Academic Editor:

Krzysztof Rogowski

Received: 7 June 2021

Accepted: 29 June 2021

Published: 30 June 2021

Publisher's Note: MDPI stays neutral with regard to jurisdictional claims in published maps and institutional affiliations.

Copyright: (c) 2021 by the authors. Licensee MDPI, Basel, Switzerland. This article is an open access article distributed under the terms and conditions of the Creative Commons Attribution (CC BY) license (https:/ / creativecommons.org/licenses/by/ $4.0 /)$.
College of Materials Science and Engineering, Nanjing Tech University, Nanjing 211816, China; machine_mouxin@163.com (X.M.); fangchao_jia@163.com (F.J.)

* Correspondence: powderfang@163.com or powder8817@njtech.edu.cn (Y.F.); ccw@njtech.edu.cn (C.C.)

\begin{abstract}
Due to the uneven materials dispersion and high dust concentration in industrial applications of turbo air classifiers, a high-efficiency rotor classifier was designed. Numerical simulations by ANSYS-Fluent 19.0, the effects of rotor cage shape, the number of blades, and the blade profile on the inner flow field, as well as classification performance, were investigated. The simulation results indicated a significant improvement in flow field distribution near the classification surface with the conical rotor cage. Furthermore, there was an average reduction of $10.1 \%$ in cut size, as well as a $23.6 \%$ increase in classification accuracy. When the number of blades was 36 , the flow field distribution between the blades was relatively uniform and a smaller cut size was obtained at a higher classification accuracy. A streamline blade with $52^{\circ}$ as the inlet installation angle effectively reduced the impact of the airflow on the blade and eliminated the inertia anti-vortex between blades. The cut size reduction was 4.7-6.3\%, with a basically unchanged classification accuracy. The material classification experimental results were in agreement with the simulated results. The discrete phase model (DPM) could well-predict the cut sizes and classification accuracy, but it could not present the fishhook effect. The present study provides theoretical guidance for the structural optimization of an air classifier with a rotor cage.
\end{abstract}

Keywords: high-efficiency rotor classifier; rotor cage shape; number of blades; blade profile; inner flow field; classification performance

\section{Introduction}

An air classifier is a key piece of equipment in the powder preparation process that is widely used in the mineral processing, food processing, and fine chemical industries [1-4]. The performance requirements for air classifiers are increasing with applications of ultra-fine powders. High-efficiency and energy-saving air classifiers are receiving widespread attention.

The turbo air classifier is a mainstream dynamic classifier for industrial applications that has a simple structure and controllable product granularity. However, with the surge in demand for powder production capacity, many problems have arisen in the industrial application of turbo air classifiers due to structural limitations [5,6]. On issue is a high dust concentration in the annular area; material classification is mainly concentrated in the annular area, so if a material cannot be sorted out in time, this would be a high dust concentration in the annular area, which would increase the chance of particle collision and reduce classification accuracy. A second problem is the uneven dispersion of materials; the number of feed inlets cannot be indefinitely increased with the increase in the demand for capacity, which has led to the uneven distribution of materials on the spreading plate and resulting in the formation of a thick material curtain, which is not conducive for material classification. A third problem is that the guide vanes can become severely worn and cannot be easily replaced. The materials in the annular area mainly rely on the impact guide blade to settle and be collected as coarse powder. The guide vanes wear out as a result and cannot be easily replaced due to structural limitations. In recent years, with 
the rapid development of computational fluid dynamics (CFD), researchers have focused more attention on the study of powder classification technology and the development of classification equipment, and they have made progress in many respects. In order to solve these problems, many scholars at home and abroad have become devoted to the research of optimizing the inner flow field distribution [7-9] and structurally improving the turbo air classifier [10-12]. However, it should be noted that most studies have focused on local optimization. The problems that have occurred in the industrial applications of turbo air classifiers have still not been positively solved. Since classification equipment is gradually moving towards high production levels and large scales, problems such as uneven material spreading and dust concentration are becoming more obvious. Therefore, in order to address these problems, a high-efficiency rotor classifier was designed. When the high-efficiency rotor classifier was used in combination with the static classifier, the above problems were effectively avoided, thereby replacing the turbo air classifier. The structure is shown in Figure 1. A static classifier for the pre-classification of materials can effectively reduce the content of coarse particles and decrease dust concentration in the classification chamber. Meanwhile, the wind feeding method allows for the material to be fully dispersed.

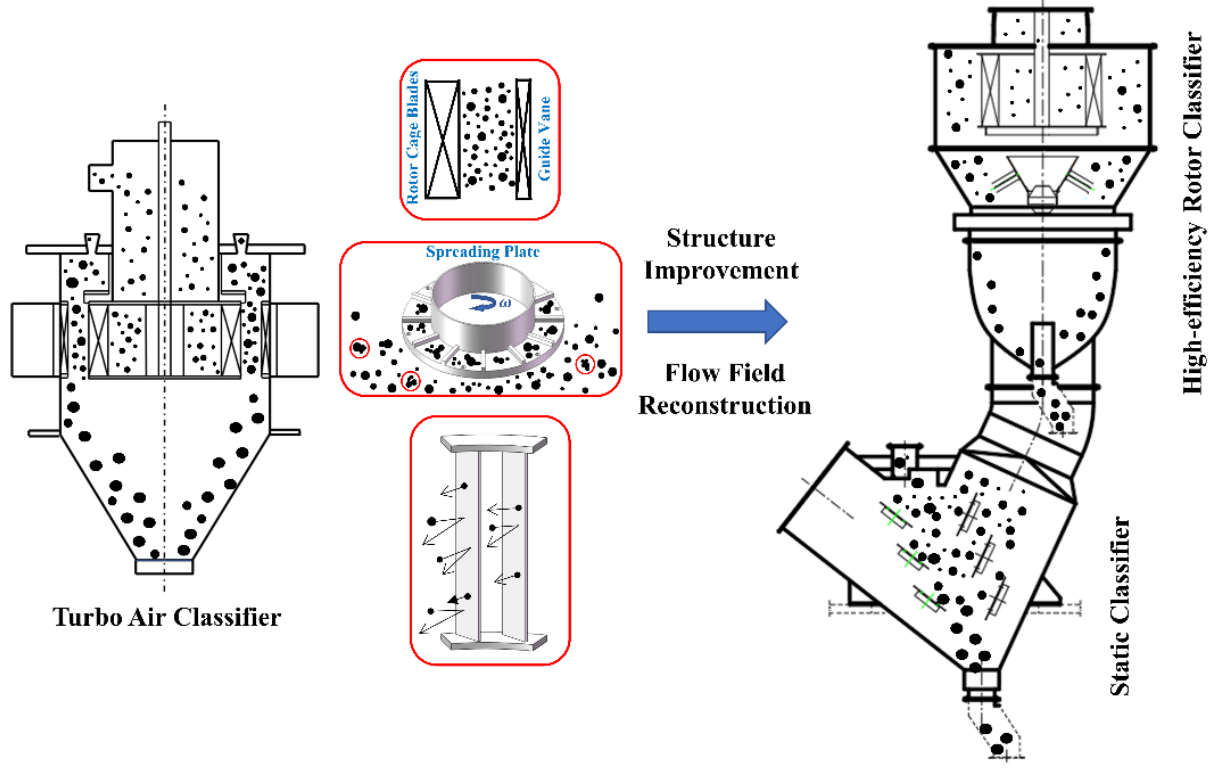

Figure 1. The turbo air classifier (left) and the new combined classifier (right).

As the core component of the classifier, the structural parameters of the rotor cage directly affect the flow field distribution and classification performance of the classifier. Hence, many researchers have focused on the rotor cage. Since material classification is mainly concentrated near the classification surface, the flow field distribution at the entrance of the rotor cage has been investigated. According to the results, maintaining the stability of the flow field on the classification surface is conducive to improving the classification performance [13-15]. The present research mainly focused on turbo air classifiers. Hence, the flow field distribution inside the classifier with a downward air inlet remains unclear. For a high-efficiency rotor classifier with a downward air inlet, further study is needed to determine how to ensure the uniformity of airflow distribution near the classifying surface. The rotor cage blade is the core part of the rotor cage, and it plays a crucial role in material classification. The blade pitch has a great influence on the pressure drop and classification efficiency of the classifier. For instance, Yu et al. [16] analyzed the effect of the inner and outer rotor cage diameters on the flow field of a turbo air classifier. The results indicated that the inner and outer diameters of the rotor cage had a significant effect on the velocity distribution in the channel. The inner and 
outer diameters of the rotor cage should not be too large or too small. Jiang et al. [17] performed numerical simulations on rotor cages with different blade pitches in order to observe the flow field changes and energy losses in the classifier. It was found that there was an optimal blade pitch in the rotor cage, where the flow field in the classifier was the most stable and the classifying performance was the best. Liu et al. [18] combined their experiments with actual production experience, and five different blade numbers were selected for the numerical analysis. According to the research, the rough running phenomenon improved as the number of blades increased, and the coarse particle content gradually decreased. However, an excessive amount of blades would increase the chances of particle and blade collision, preventing the particles from being sorted out in time. Hence, a reasonable number of blades is conducive to improving the classification performance of air classifiers. As a necessary path in the material sorting process, the flow field distribution in the rotor cage channel directly determines classification efficiency $[19,20]$. In order to improve the flow field distribution in the rotor cage channel, some researchers have made improvements to the blade profile. The results have indicated that a positively bended arc blade can significantly improve the flow field distribution in turbo air classifiers, reducing the inertial rotating vortex and radial velocity fluctuation between the blades [21-24]. The Tianjin Cement Industry Design and Research Institute carried out numerical simulations of pressure loss and classification efficiency for blades widely used in the industry, and the researchers proposed a new type of $U$-shaped blade classifier with low pressure loss and high efficiency [25]. However, most previous studies have been limited to specific equipment, and the designed blades have not been universally applicable. On the contrary, when using the flow field distribution to determine the inlet and outlet installation angle, using the single arc method to design the blade can be suitable for different equipment.

In the present study, the numerical simulation of a high-efficiency rotor classifier for industrial applications was conducted with the aid of the ANSYS-Fluent 19.0 software. The effects of rotor cage shape, the number of blades, and the blade profile on the inner flow field, as well as the classification performance of the classifier, were investigated. According to the results, the conical rotor cage was more suitable for the classifier in the form of a downward air inlet. An optimum number of blades existed for the conical rotor cage. The designed streamlined blade could effectively improve the flow field distribution between the blades. Furthermore, the simulation results were verified by powder classification experiments. The present study not only designed a high-efficiency rotor classifier but also provided a new strategy for the design and optimization of rotor cages.

\section{Apparatus Description}

\subsection{Structural Characteristics}

The performance of an air classifier mainly depends on the two stages of material dispersion and classification, and the organic combination of these two is the key to optimize the design of such a classifier [26]. Hence, a high-efficiency rotor classifier was designed with the full consideration of material dispersion and classification. The structural characteristics are as follows:

(1) The adequate dispersion of the material is a prerequisite to ensure high-efficiency classification. Therefore, the traditional approach of spreading the material was abandoned and replaced by wind feeding, and the material was carried into the highefficiency rotor classifier by airflow. The wind feeding method made the material fully mixed with the airflow, thus ensuring the dispersion of the material.

(2) The wind feeding method decreased the coarse particle content and fully dispersed the material. This is conducive to the timely sorting out of material particles and the effective reduction of the dust concentration in the classifying chamber.

(3) The material classification effect directly determines classification efficiency and energy consumption. Due to its stability and controllability, the centrifugal field was chosen as the classification force field. 
(4) In the high-efficiency rotor classifier, the guide vanes were removed, and the conical rotor cage was chosen to strengthen and stabilize the centrifugal classification force field. The swirling flow and tangential shear force generated by the rotation of the conical rotor cage increased the material dispersion performance and improved the classification efficiency.

(5) The spindle in a conventional turbo air classifier not only needed to drive the rotation of the rotor cage but also dispersed the material. As a comparison, the spindle in the high-efficiency rotor classifier only needed to drive the rotation of the rotor cage, which effectively reduced energy consumption.

\subsection{Working Principle}

The working principle for the high-efficiency rotor classifier is presented in Figure 2. After the milled material is dispersed and pre-classified by the static classifier, the coarse material is returned to the mill, while the fine material is carried into the high-efficiency rotor classifier with the airflow. As the airflow moves to the cone part, the flow velocity decreases due to the sudden increase in the cross-section of the channel. Coarse particles are collected as coarse powder, since these bounce back from the collision with the guide cone under inertia. The remaining material continues to be carried by the airflow through the guide cone rectification into the classifying chamber. In the classifying chamber, the material particles are mainly subjected to the air drag force and the centrifugal force generated by the rotor cage. The coarse particles are thrown out of the rotor cage due to the large centrifugal force, hit the wall, lose their kinetic energy, fall down, and are collected as coarse powder. The fine particles are carried by the airflow through the classifying surface into the rotor cage, discharged from the fine powder outlet, and collected as finished products.

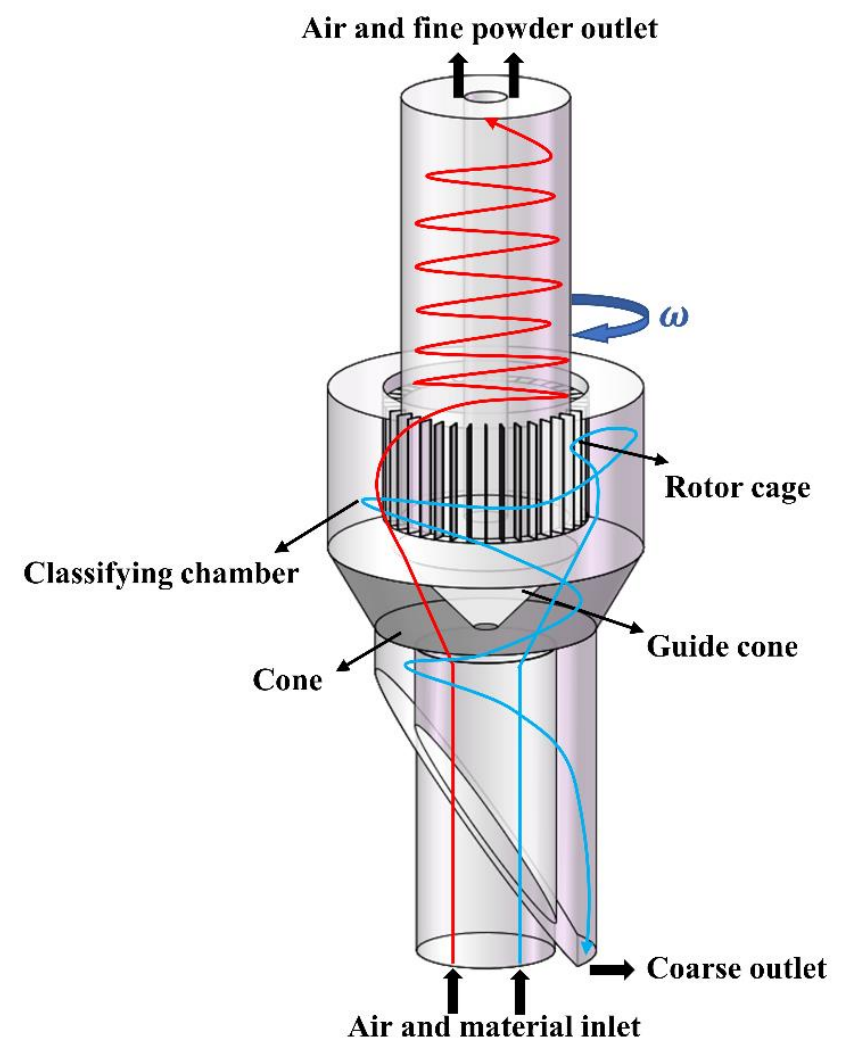

Figure 2. Working principle of the high-efficiency rotor classifier. 


\section{Model Description and Boundary Conditions}

\subsection{Geometry Modeling and Meshing}

The modeling for the computational model of the high-efficiency rotor classifier was performed using SolidWorks. The main structural dimensions of the classifier are presented in Figure 3. The dimensions for the blades were $20 \mathrm{~mm}$ in length, $2 \mathrm{~mm}$ in thickness, and $150 \mathrm{~mm}$ in height, and the blades were evenly distributed along the outer edge of the rotor cage. In order to avoid backflow during the calculation, the central outlet pipe was extended up to three times of the diameter of the pipe. The computational model was divided into six regions, and each region was meshed using ICEM CFD in order to ensure the quality of the mesh. Except for the coarse powder outlet area, in which an unstructured mesh was used, a structured mesh was used for all other areas, and these areas were connected through the interface.

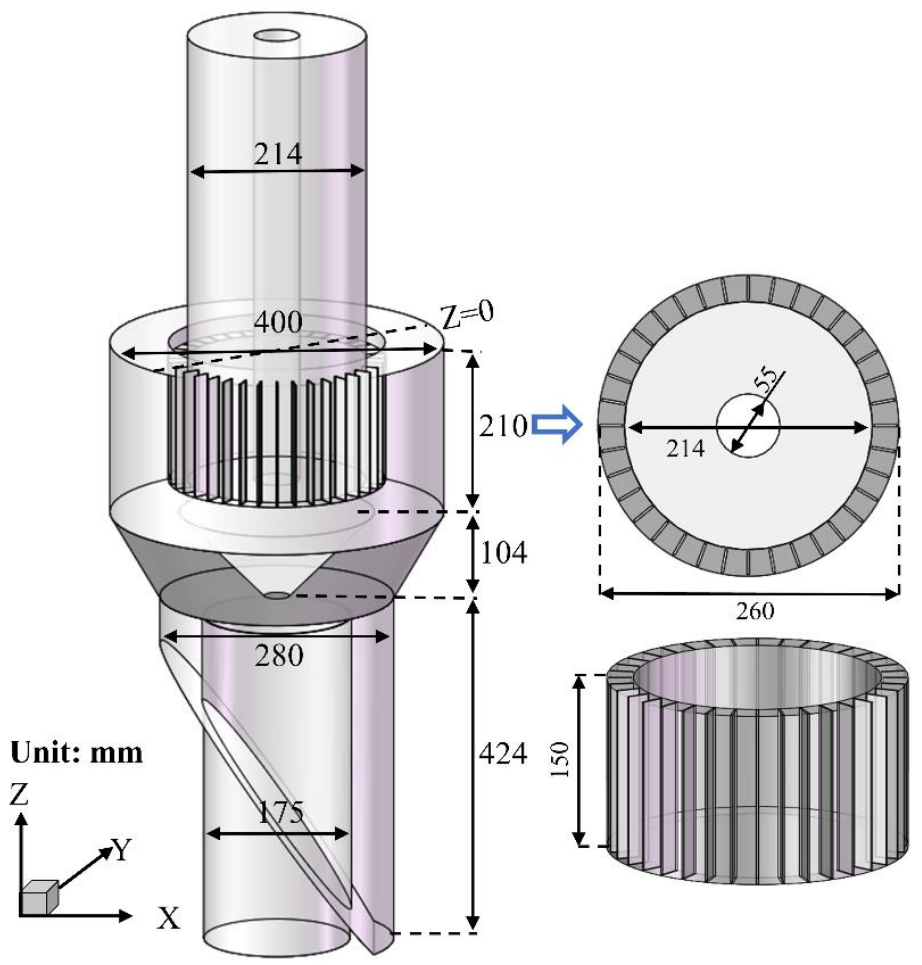

(a) view

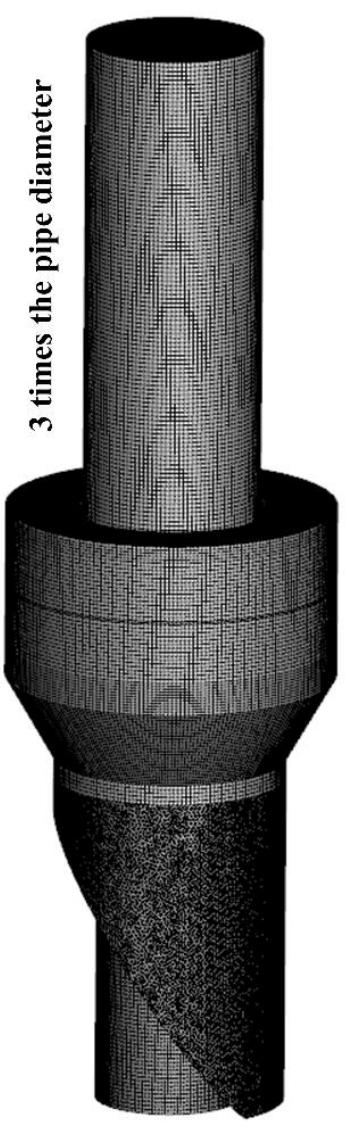

(b) mesh

Figure 3. High-efficiency rotor classifier structural dimensions and the CFD mesh.

In order to verify the mesh independence, the average radial velocity of the airflow at the entrance of the rotor cage with different mesh numbers was analyzed and compared. The results are presented in Table 1. When the number of meshes exceeded 2.02 million, there was no significant difference in the average radial velocity. Hence, in consideration of computation time and accuracy, the 2.02 million mesh model was chosen for the simulation. The number of grids in each region is shown in Table 2.

Table 1. Influence of the number of meshes on average radial velocity at the entrance of the rotor cage.

\begin{tabular}{cccccc}
\hline Mesh Number (million) & 101.7 & 148.2 & 202.1 & 256.2 & 301.4 \\
\hline Average Radial Velocity (m/s) & -2.67 & -2.93 & -3.28 & -3.30 & -3.22 \\
\hline
\end{tabular}


Table 2. Mesh number in each region of the high-efficiency rotor classifier.

\begin{tabular}{cc}
\hline Region & Mesh Number \\
\hline Fine powder outlet & 329,840 \\
Rotor cage & 226,894 \\
Classifying chamber & 384,791 \\
Guide cone region & 256,790 \\
Air inlet & 353,760 \\
Coarse powder outlet & 468,462 \\
Sum & $2,020,537$ \\
\hline
\end{tabular}

\subsection{Boundary Conditions and Parameter Settings}

The boundary conditions were set by combining the structural characteristics and flow field characteristics of the high-efficiency rotor classifier. The SIMPLEC algorithm was adopted for pressure-velocity coupling, and the QUICK difference scheme was used for convective diffusion. The no-slip boundary conditions were used for the wall boundary, and the near-wall surface was treated with standard wall functions. The simulation working conditions were set as follows: an air volume of $1200 \mathrm{~m}^{3} / \mathrm{h}$, rotation speed of $500 \mathrm{rpm}$, and feeding speed of $650 \mathrm{~kg} / \mathrm{h}$. The inlet was defined as a velocity inlet with a flow velocity of $13.8 \mathrm{~m} / \mathrm{s}$, assuming that the airflow was uniformly distributed in the inlet cross-section and direction perpendicular to the cross-section. The outlet was defined as a fully developed pipe flow (outflow). The multiple reference coordinates system model (MRF) was adopted to simulate the rotational motion of the rotor cage in the classification chamber, with the direction of rotation was defined as clockwise. The rotor cage blades were set to 'moving wall,' with a rotational speed of zero relative to the rotating area.

The discrete phase model (DPM) was adopted to simulate the trajectory of the particles. In order to simplify the model, the coarse powder outlet and sloping surface on the right side were set to 'trap.' This means that the calculation would be aborted if the material particles touched this surface, and they were collected as coarse powder. Considering the interaction between particles and the discrete vortex, the discrete random walk model (DRW) was used to simulate the dispersion effect of the turbulent flow on particles. Since material particles above $120 \mu \mathrm{m}$ were already sorted out in the pre-classification stage, merely the classification efficiency of material particles within $0-120 \mu \mathrm{m}$ needed to be discussed. In the velocity inlet for establishing 60 groups of surface injection source, the density of the material particles was set at $2750 \mathrm{~kg} / \mathrm{m}^{3}$ of calcium carbonate, and the particle size distribution was set to $0-120 \mu \mathrm{m}$. The tromp curves were obtained by tracking the particle trajectory, and the ratio of the number of particles trapped (trap) to the total number of particles tracked (track) was taken as the classification efficiency.

\subsection{Mathematical Models}

\subsubsection{Turbulence Model}

The three-dimensional steady simulation was performed using ANSYS-Fluent 19.0 (Canonsburg, PA, USA). For the case of incompressible flow, the mass and momentum equations are as follows:

$$
\begin{gathered}
\frac{\partial u_{i}}{\partial x_{i}}=0 \\
\rho u_{j} \frac{\partial u_{i}}{\partial x_{j}}=-\frac{\partial p}{\partial x_{i}}+\frac{\partial}{\partial x_{j}}\left[\mu\left(\frac{\partial u_{i}}{\partial x_{j}}+\frac{\partial u_{j}}{\partial x_{i}}\right)\right]-\rho \frac{\partial \overline{u_{i}^{\prime} u_{j}^{\prime}}}{\partial x_{j}}
\end{gathered}
$$

where $\rho$ refers to the fluid density, $u_{i}$ refers to the fluid velocity, $x_{i}$ refers to the position, $\mu$ refers to the fluid viscosity, $p$ refers to the static pressure, and $-\rho \frac{\partial \overline{u_{i}^{\prime} u_{j}^{\prime}}}{\partial x_{j}}$ refers to the Reynolds stress term.

Considering the calculation time and accuracy, the Reynolds stress model (RSM) was chosen for the turbulence model. The model used the transport equation to solve the 
Reynolds stress, according to the law of time homogenization, in order to better predict the turbulent flow in the air classifier. The prediction of the partial classification efficiency and pressure drop also agreed well with the experimental data $[2,27,28]$. The transport equations for the transport of the Reynolds stresses may be written as follows:

$$
\frac{\partial\left(\rho u_{k} \overline{u_{i}^{\prime} u_{j}^{\prime}}\right)}{\partial x_{k}}=\left(D_{T, i j}+D_{L, i j}\right)+P_{i, j}+G_{i, j}+\varphi_{i, j}+\varepsilon_{i, j}+F_{i, j}+S_{u s e r}
$$

where $\rho$ refers to the fluid density, $u_{i}^{\prime}$ refers to the fluctuating velocity to the direction $i$, $x_{k}$ refers to the positional length, $D_{i j}$ refers to the diffusion term, $P_{i j}$ refers to the stress production term, $G_{i j}$ refers to the buoyancy production term, $\varphi_{i j}$ refers to the pressure strain term, $\varepsilon_{i j}$ refers to the dissipation term, $F_{i j}$ refers to the production by system rotation term, and $S_{\text {user }}$ refers to the user-defined source term. Since the fluid was considered incompressible, Equation (3) with $G_{i j}=0$. The RSM model required the following empirical constants: $\sigma_{k}=0.82, \sigma_{\varepsilon}=1.00, C_{\varepsilon 1}=1.44, C_{\varepsilon 2}=1.92$.

\subsubsection{Discrete Phase Model}

Since the volumetric amount of the inlet particles was below the traditional dilute limit of $0.1 \%$, a one-way coupling was assumed [29]. Hence, the interaction between particles and the effect of particles on the gas phase were neglected. In the Lagrangian coordinate system, the DPM predicted the trajectory of the particle based on the forces on the particle, and the equation for motion was derived as follows:

$$
m_{p} \frac{d \overrightarrow{u_{p}}}{d t}=m_{p} \frac{\vec{u}-\overrightarrow{u_{p}}}{\tau_{r}}+m_{p} \frac{\vec{g}\left(\rho_{p}-\rho\right)}{\rho_{p}}+\vec{F}
$$

where $m_{p}$ refers to the particle mass, $t$ refers to the time, $\vec{u}$ refers to the fluid velocity, $\overrightarrow{u_{p}}$ refers to the particle velocity, $\vec{g}$ refers to the gravitational acceleration, $\rho$ refers to the fluid density, $\rho_{p}$ refers to the particle density, $\vec{F}$ refers to the additional force, $m_{p} \frac{\vec{u}-\overrightarrow{u_{p}}}{\tau_{r}}$ refers to the drag force, and $\tau_{r}$ refers to the relaxation time of the particle, which can be calculated using the following equation:

$$
\tau_{r}=\frac{\rho_{p} d_{p}^{2}}{18 \mu} \frac{24}{C_{d} R e}
$$

where $\mu$ refers to the molecular viscosity of the fluid, $d_{p}$ refers to the particle diameter, and Re refers to the relative Reynolds number, which was derived as follows:

$$
\operatorname{Re}=\frac{\rho d_{p}\left|\overrightarrow{u_{p}}-\vec{u}\right|}{\mu}
$$

\subsection{Experimental Setup}

The scheme for the experimental setup is presented in Figure 4. The experimental setup mainly comprised the high-efficiency rotor classifier, cyclone collector, pulse bag filter, high-pressure induced draft fan, and control system. The analysis of the particle size distribution of the samples was performed using the Mastersizer 2000 laser particle sizer. The classifier pressure drop $(\Delta \mathrm{P})$ was calculated by the average inlet static pressure minus the average outlet static pressure, which could be measured using a U-type manometer. 


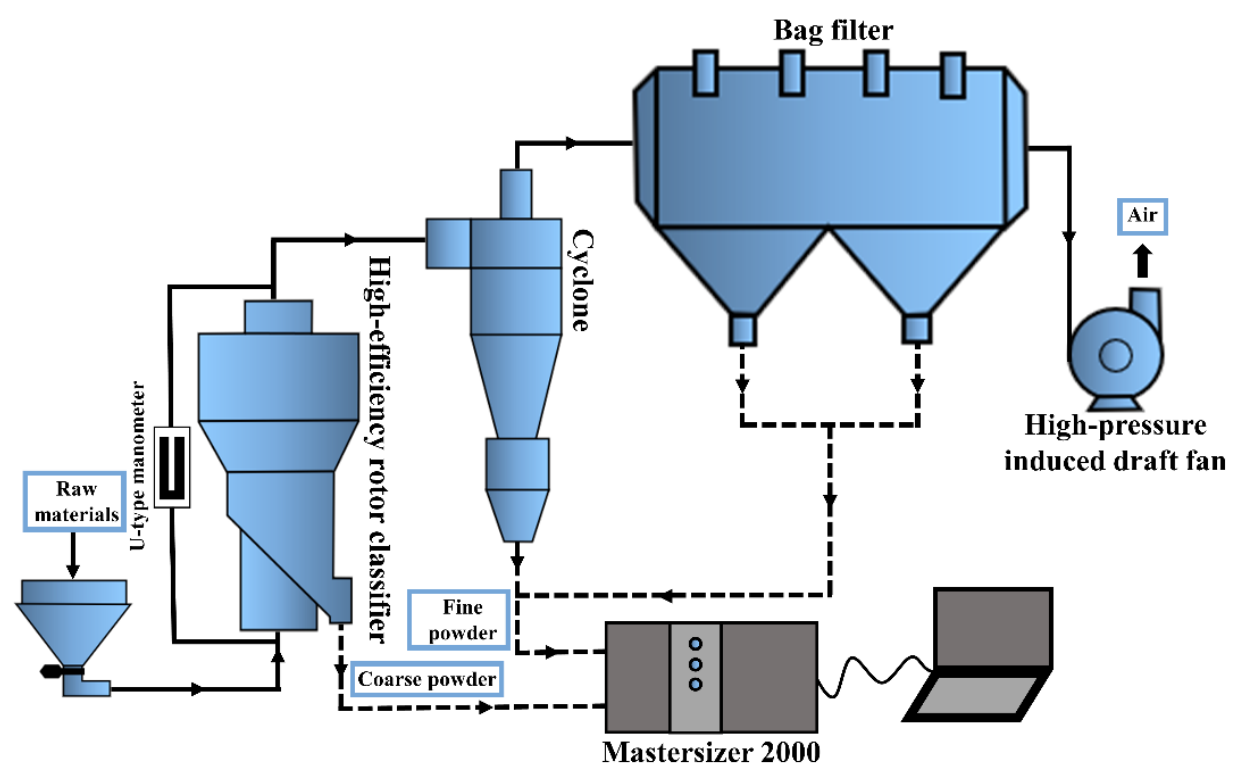

Figure 4. Scheme for the experimental setup.

The evaluation of the performance of the high-efficiency rotor classifier was performed using parameters, such as cut size $\left(\mathrm{d}_{50}\right)$, classification accuracy $(\mathrm{K})$, and pressure drop $(\Delta \mathrm{P})$, which were obtained from simulations and experiments. The characteristic particle sizes, such as $d_{50}, d_{25}$, and $d_{75}$, could be obtained by partial classification efficiency curves. Then, $K$ was calculated according to the formula $K=d_{25} / d_{75}$. A lower value for $d_{50}$ and a higher value for $\mathrm{K}$ indicate better classification performance.

\section{Simulation Results and Analysis}

\subsection{Effect of the Rotor Cage Shape on Velocity Distribution near the Classification Surface}

In order to clarify the distribution characteristics of the flow field in the classifier, multiple cross-sections were selected as the research object in the classifier, and velocity cloud diagrams and local vector diagrams of different cross-sections were obtained, as shown in Figure 5. It can be observed from the figure that the airflow entered from the air inlet, entered the classification chamber through the guide cone, entered the rotor cage under the action of the central negative pressure, and finally discharged from the fine powder outlet. The velocity reached the maximum at the outlet of the fine powder, which was favorable to the output of fine powder, while the velocity was smaller in the near-wall area of the classification chamber, which was favorable to the fall of the coarse powder. According to the velocity vector diagram, it can be observed that after the air flow was rectified by the guide cone, most of the air flow smoothly entered the rotor cage and a small part of the air flow continued to move in the axial direction. Inside the rotor cage, the airflow moved upward at an angle of approximately $60^{\circ}$, causing part of the airflow to collide with the inner wall at the bottom of the rotor cage and deflect to form a vortex. This seriously affected the conveyance and classification of the material, which in turn compressed the effective classification area. While outside the rotor cage, the airflow continued to move in the axial direction, colliding with the wall at the top of the classification chamber and turning to form a vortex. Then, the vortex formed the secondary scouring for the material after classification, reducing the chance for fine particles to be collected as coarse powder. 


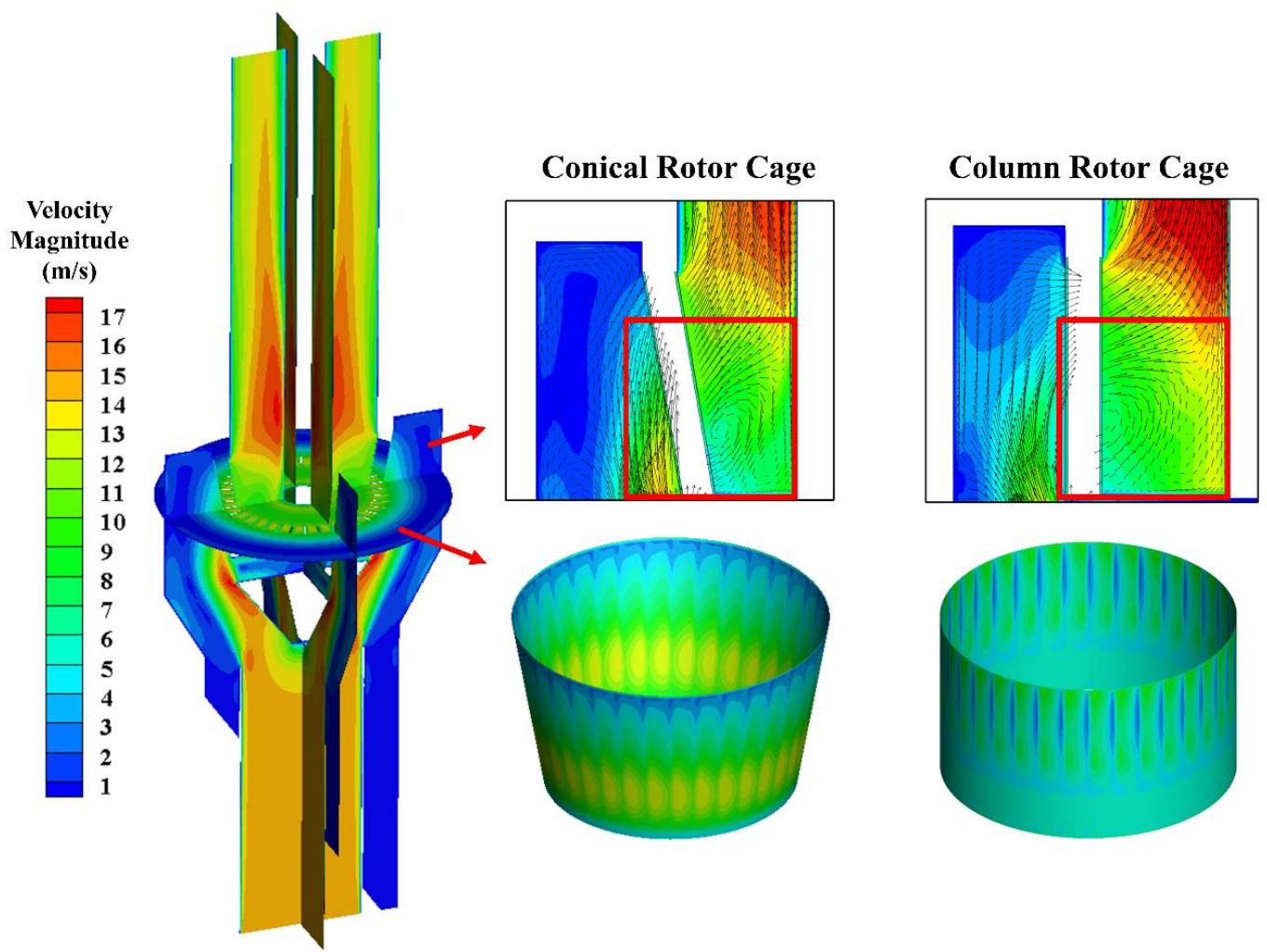

Figure 5. Cloud diagram for the high-efficiency rotor classifier with different cross-sectional velocities and local velocity vectors.

In order to obtain a higher classification accuracy and efficiency, the "aerodynamic screen" must maintain the same mesh size, that is, to ensure a stable and evenly distributed airflow near the classification surface [6]. According to the flow field distribution characteristics, since the high-efficiency rotor classifier was combined with the downward air inlet form, it remained difficult to ensure that the velocity distribution near the classification surface remained consistent along the axial direction. In order to determine the suitable rotor cage shape for the high-efficiency rotor classifier, the velocity distributions near the classifying surface of the column rotor cage and conical rotor cage were analyzed and compared.

The outer surface of the rotor cage was taken as the object of study. The radial and tangential velocity distributions of the airflow were obtained by taking points along the axial direction at the entrance of the rotor cage, as shown in Figure 6. The cloud diagram for the radial and tangential velocity distributions corresponded to different rotor cage shapes, as shown in Figure 7. From the figure, it can be observed that the radial and tangential velocities of the conical rotor cage decreased to different degrees, when compared to the column rotor cage, and that the velocity distribution was more uniform with smaller gradients. The decreases in radial and tangential velocities were mainly correlated to the rotor cage shape. Since the column rotor cage was combined with the downward air inlet form, most of the airflow moved to the upper part of the rotor cage. Meanwhile, a large vortex was formed at the bottom of the rotor cage, which made it difficult for the airflow to evenly enter the rotor cage. Hence, the radial velocity of the airflow presented a distribution of upper large and lower small. Influenced by the vortex, the tangential velocity of airflow also had a large velocity gradient. However, the conical rotor cage was combined with the downward air inlet form, which increased the cross-sectional area at the entrance of the rotor cage due to the structural characteristics of large upper and small lower, resulting in a decrease in radial velocity. The vortex at the bottom of the rotor cage was weakened by the structural limitation, thus decreasing the tangential velocity at the bottom of the rotor cage. Since the conical rotor cage was more catered to the airflow 
direction, the airflow could evenly enter the rotor cage, making the velocity distribution more uniform. The tangential velocity of the airflow at the entrance of the rotor cage was small, primarily because the tangential velocity of the airflow was mainly generated by the rotation of the rotor cage. However, as the airflow entered the rotor cage channel, the tangential velocity gradually increased until it remained stable. Hence, compared with the column rotor cage, the conical rotor cage made the flow field near the classifying surface more evenly distributed and the classifying force field more stable. Therefore, material particles were basically subjected to the same force at any position near the classification surface, which was conducive to improving the classification performance.
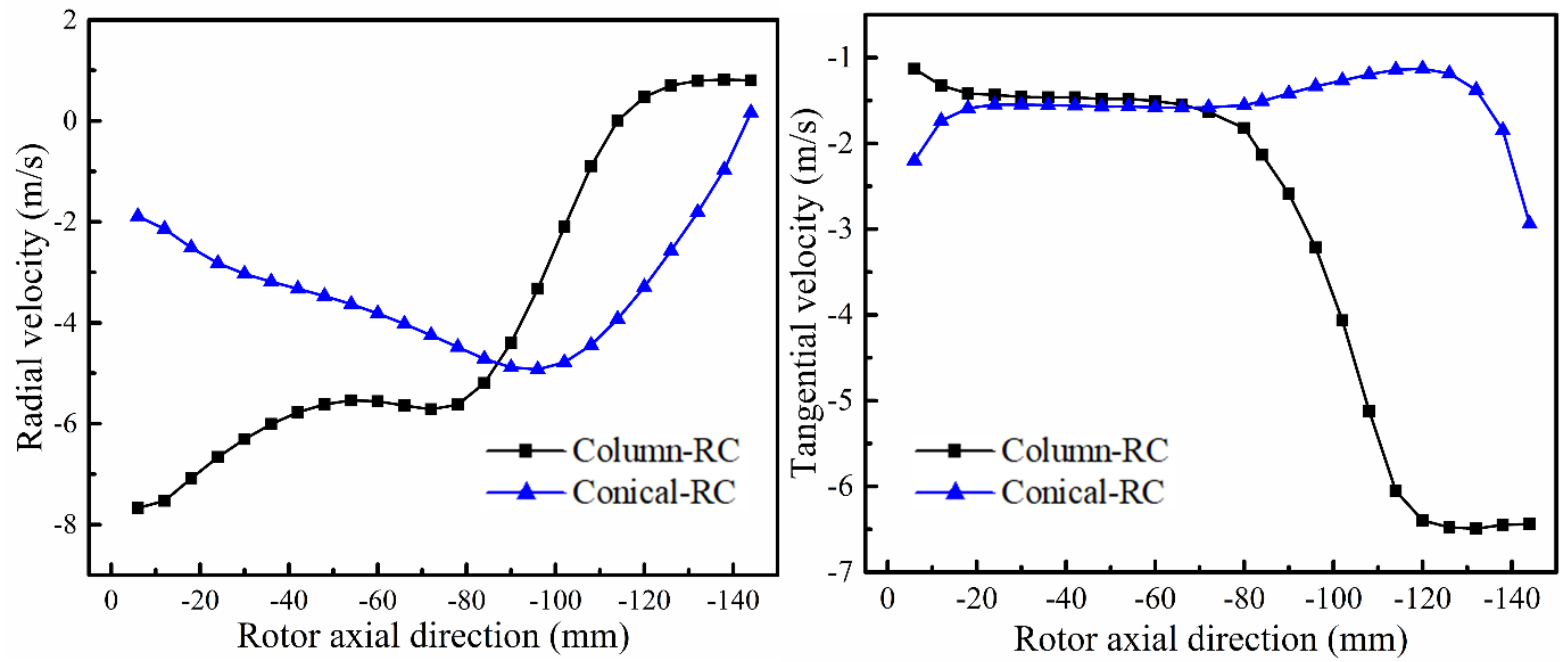

Figure 6. The radial and tangential velocity distributions of airflow at the rotor cage entrance.
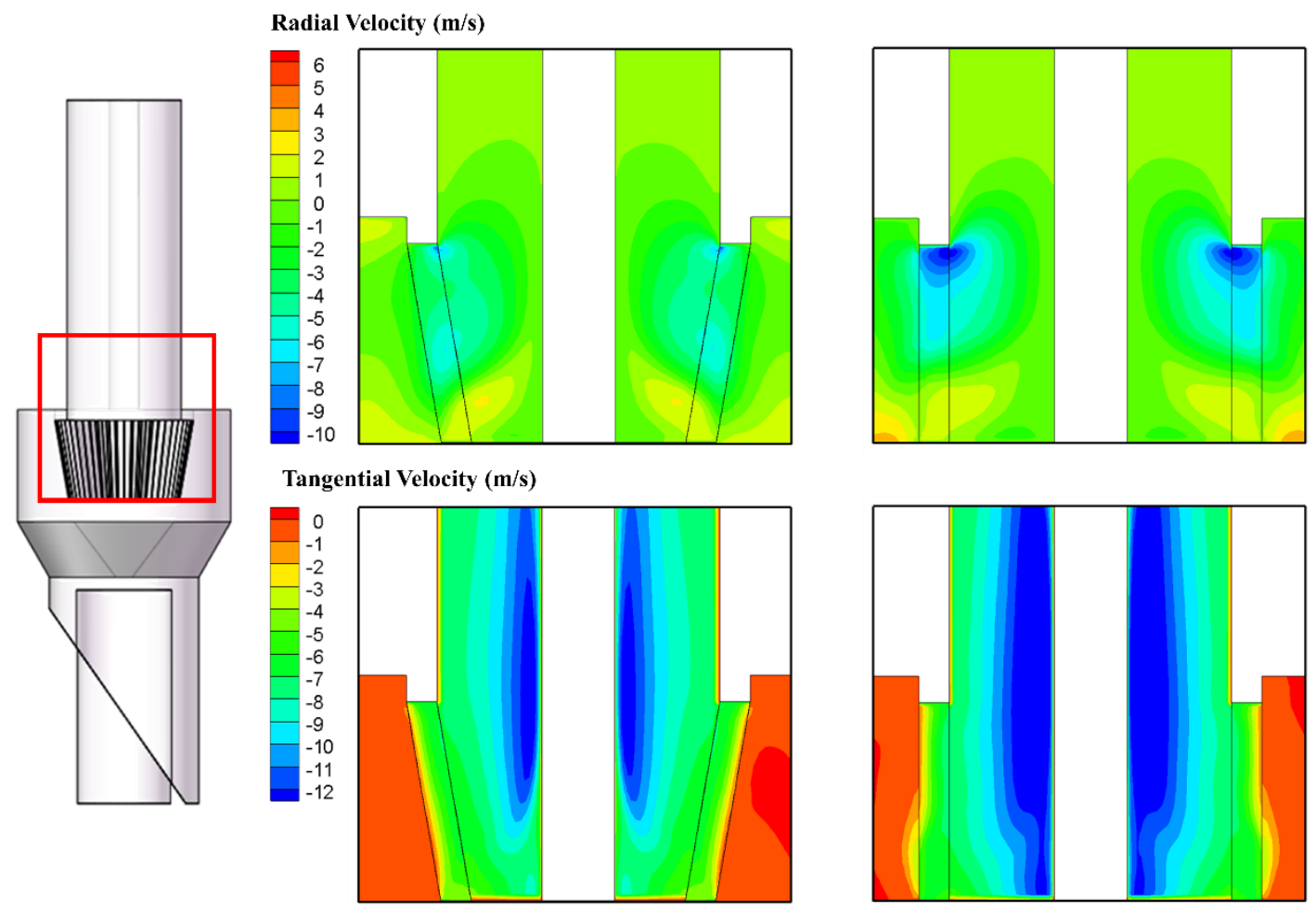

Figure 7. The cloud diagram for the radial and tangential velocity distributions corresponding to the different rotor cage shapes. 
The results for the discrete phase simulation using the column and conical rotor cage at different operating conditions are presented in Table 3. For each operation condition, the cut size using the column rotor cage was smaller than that using the conical rotor cage, with a decrease of $9.2-11.3 \%$. In addition, $\mathrm{K}$ increased by $13.0-25.9 \%$. According to the simulation results, it could be observed that the conical rotor cage could effectively improve the classification performance and had a better adaptability to different working conditions.

Table 3. Comparison of the cut size $\left(\mathrm{d}_{50}\right)$ and classification accuracy $(\mathrm{K})$ for the column and conical rotor cage.

\begin{tabular}{cccccc}
\hline \multirow{2}{*}{$\mathbf{v}(\mathbf{m} / \mathbf{s})$} & $\mathbf{n}(\mathbf{r p m})$ & \multicolumn{2}{c}{ Cut Size $\left(\mathbf{d}_{\mathbf{5 0}}\right)$} & \multicolumn{2}{c}{ Classification Accuracy $(\mathbf{K})$} \\
\cline { 3 - 6 } & & Column RC & Conical RC & Column RC & Conical RC \\
\hline 13.8 & 500 & 33.6 & 29.7 & 0.54 & 0.68 \\
13.8 & 600 & 28.1 & 25.5 & 0.62 & 0.73 \\
16.2 & 500 & 38.6 & 35.0 & 0.48 & 0.61 \\
\hline
\end{tabular}

\subsection{Effect of Blade Number on Classification Performance}

The number of rotor cage blades is the key factor that affects the flow field between blades. When the number of blades increased and made the rotor cage channel narrower, the vortex intensity between blades was weakened and the flow field became more stable. However, an excessive number of blades could lead to a very narrow channel in the rotor cage, which would affect the material passage and thereby reduce the processing capacity of the classifier. Therefore, it is essential to select a reasonable number of rotor cage blades in real applications.

In order to investigate the effect of the number of rotor cage blades on classification performance, five different rotor cages with $\mathrm{N}=24, \mathrm{~N}=36, \mathrm{~N}=48, \mathrm{~N}=60$, and $\mathrm{N}=72$ blade numbers were simulated and the radial velocity distribution cloud was obtained, as shown in Figure 8. Due to the existence of vortices at the bottom of the rotor cage, only the cross-section within the effective classification area needed to be analyzed. Therefore, the velocity distributions at an XOY-plane with $\mathrm{Z}=-37.5 \mathrm{~mm}$ and $\mathrm{Z}=-75 \mathrm{~mm}$ were discussed as follows.

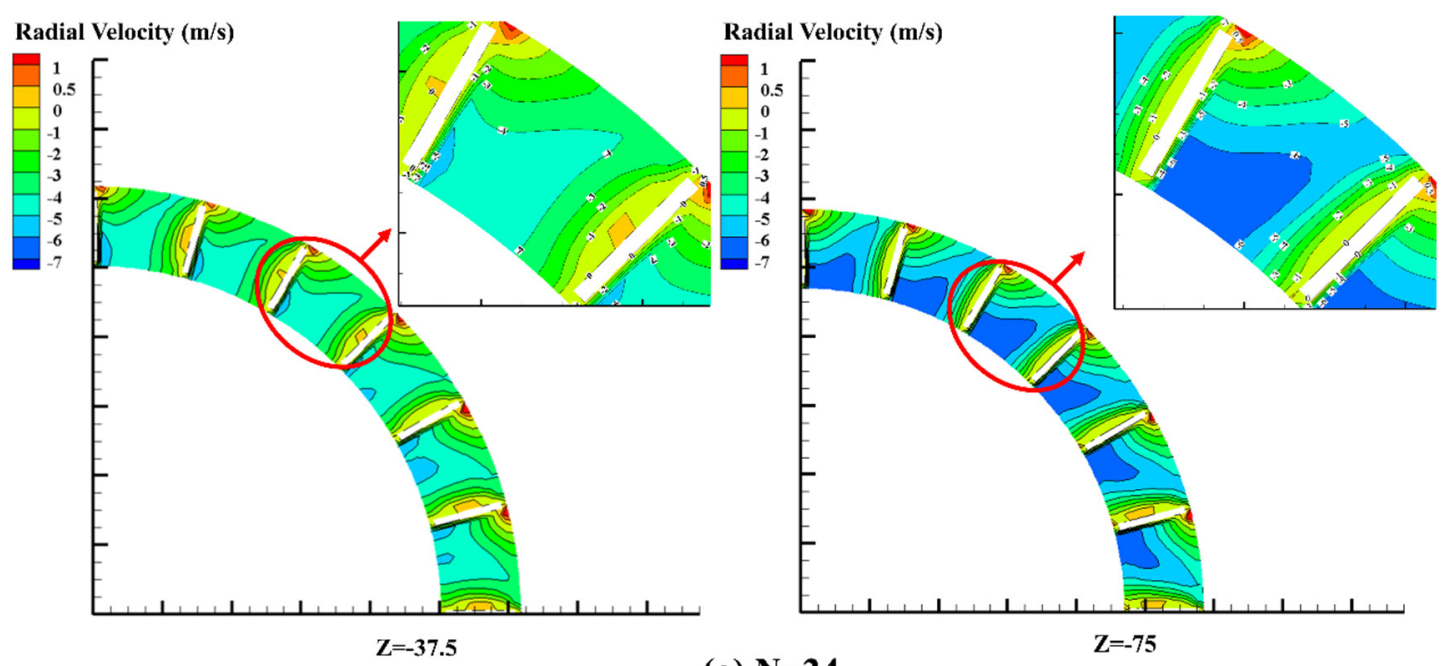

(a) $\mathrm{N}=\mathbf{2 4}$

Figure 8. Cont. 

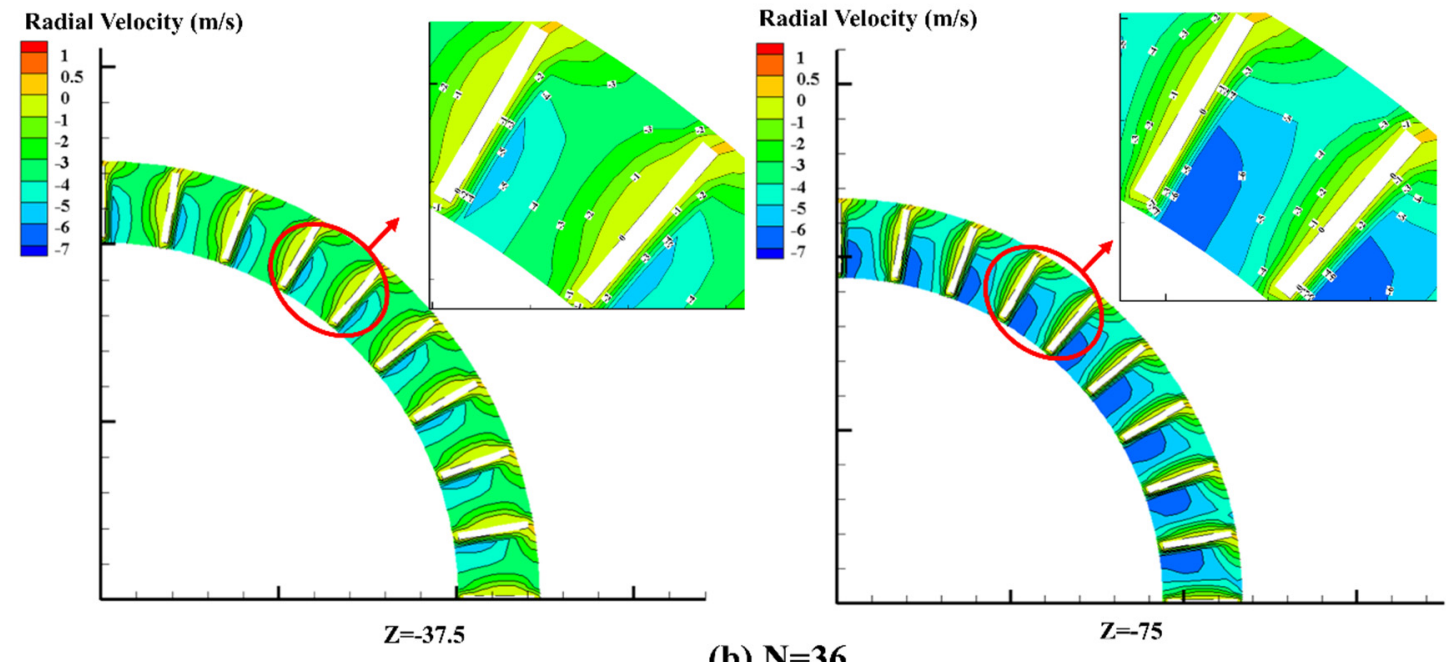

(b) $\mathrm{N}=\mathbf{3 6}$
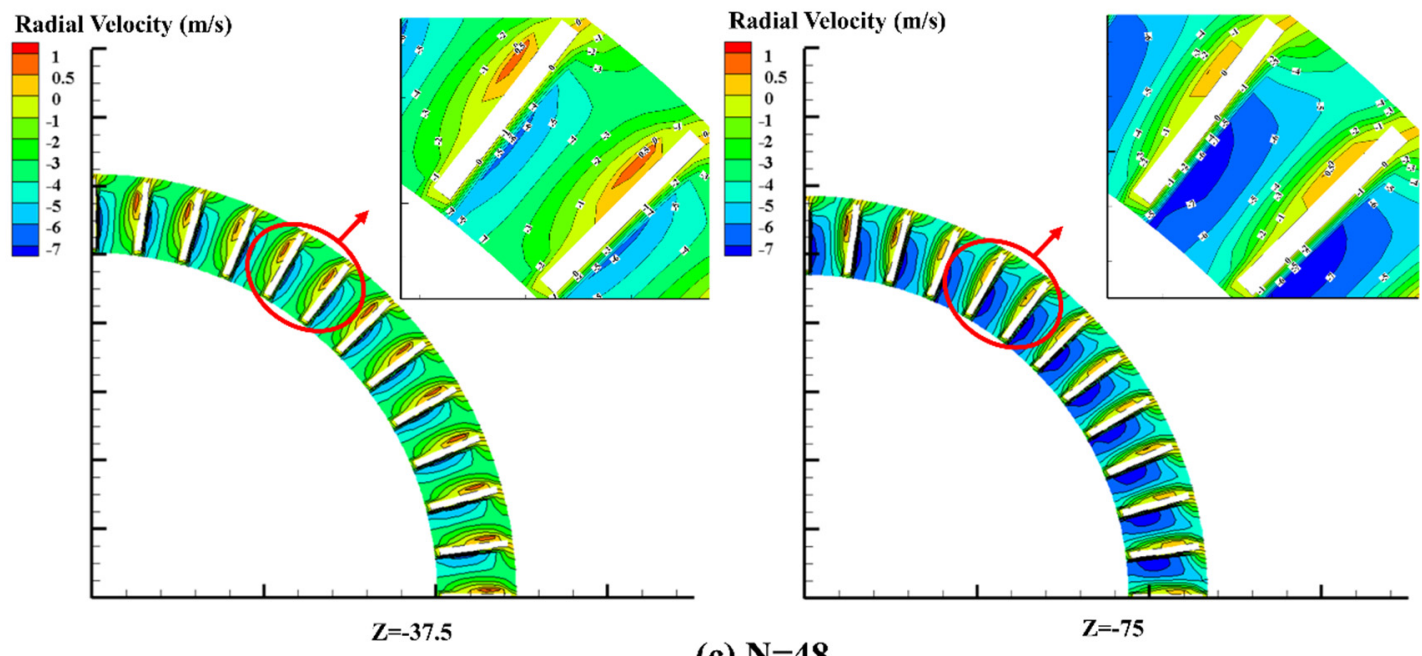

(c) $\mathrm{N}=48$
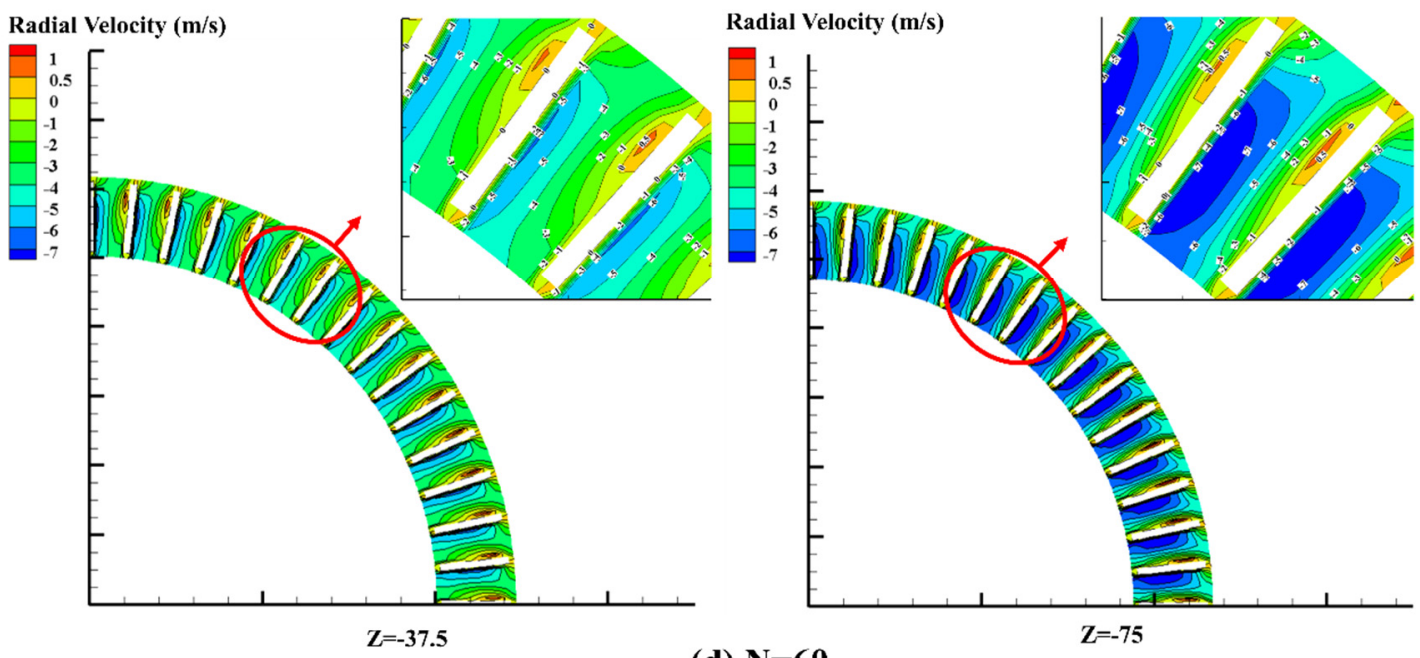

(d) $\mathrm{N}=60$

Figure 8. Cont. 

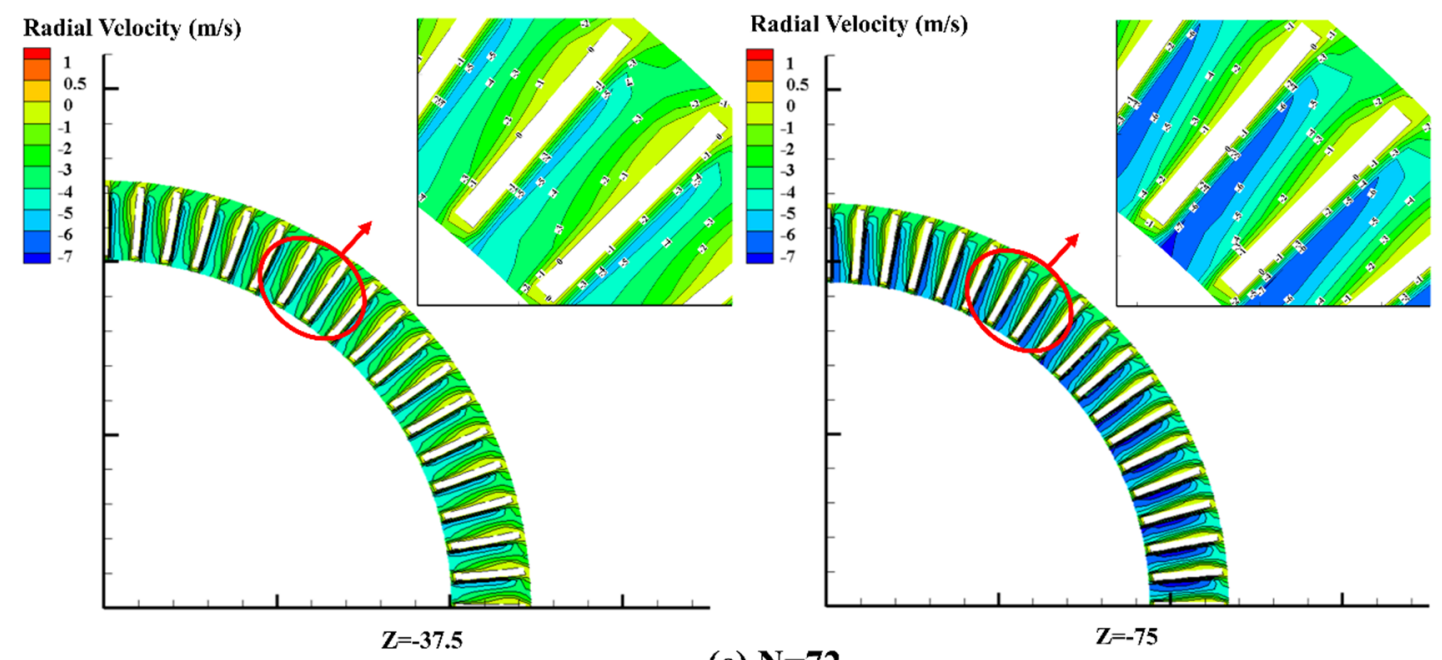

(e) $\mathrm{N}=72$

Figure 8. The cloud diagram for the radial velocity distribution corresponding to the different numbers of blades.

As shown in the figure, the radial velocity between blades was distributed in a fusiform stepped manner, and a large velocity gradient generally existed. Mainly due to the limited number of rotor cage blades, the airflow in the channel was not completely in accordance with the movement of the blade profile. There was a relative axial rotational motion in addition to the relative motion between blades in the radial direction, and these two movements compounded the relative motion of the airflow in the channel. On the side of the blade proceeding surface, two kinds of motion in the same direction superimposed on each other, making the relative velocity increase. On the side of the blade receding surface, these two motions were in opposite directions, and these canceled each other out, thus causing the relative velocity to decrease. When $\mathrm{N}<36$, the blade spacing was larger. Furthermore, in the receding surface and the rotor cage inlet, there were different degrees of anti-vortex, and the flow field between blades was unstable. When $\mathrm{N}>36$, the blade spacing gradually decreased as the number of blades increased. Furthermore, the radial velocity increased and the velocity gradient in the channel became larger. Meanwhile, the increased pressure difference between the blades made the airflow on the receding side reverse, forming an inertial anti-vortex in the channel. When the number of blades was increased to 72 , the rotor cage channel narrowed, the space for airflow movement was reduced, and the inertial anti-vortex between the blades was basically eliminated. However, excessively narrow channels would lead to an increased chance of collision between materials and blades, which would not be conducive for classification. When $\mathrm{N}=36$, the velocity gradient between blades was small, the radial velocity direction was basically the same, and the flow field was relatively stable.

According to the flow field distribution, it was observed that the flow field between the blades was relatively stable and uniform when $\mathrm{N}=36$. For further validation, the partial classification efficiency of the classifier was calculated by the DPM model. At different operating conditions, the partial classification efficiency curves that corresponded to different numbers of blades were obtained, as shown in Figure 9. The cut size and classification accuracy were calculated according to the partial classification efficiency curve, as shown in Table 4. 

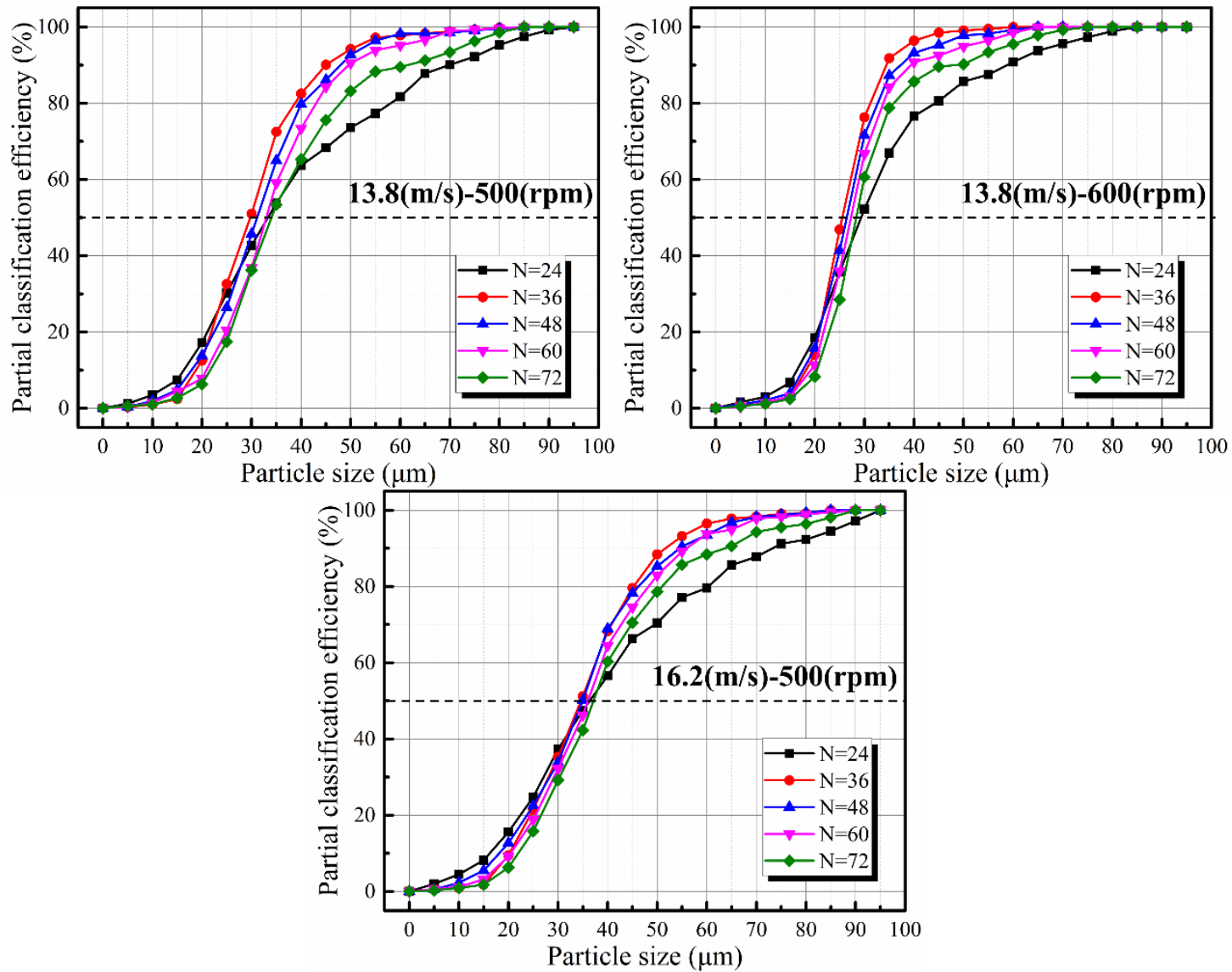

Figure 9. The classification efficiency curves corresponding to the different numbers of blades in different operating conditions.

Table 4. The cut size and classification accuracy for the different numbers of blades.

\begin{tabular}{|c|c|c|c|c|c|c|}
\hline \multirow{2}{*}{$\begin{array}{l}\text { Number of } \\
\text { Blades }\end{array}$} & \multicolumn{2}{|c|}{$13.8-500$} & \multicolumn{2}{|c|}{$13.8-600$} & \multicolumn{2}{|c|}{$16.2-500$} \\
\hline & Cut Size $\left(d_{50}\right)$ & $\begin{array}{l}\text { Classification } \\
\text { Accuracy (K) }\end{array}$ & Cut Size $\left(d_{50}\right)$ & $\begin{array}{l}\text { Classification } \\
\text { Accuracy (K) }\end{array}$ & Cut Size $\left(d_{50}\right)$ & $\begin{array}{l}\text { Classification } \\
\text { Accuracy (K) }\end{array}$ \\
\hline$N=24$ & 33.2 & 0.44 & 29.4 & 0.56 & 36.1 & 0.43 \\
\hline$N=36$ & 29.7 & 0.68 & 25.5 & 0.73 & 35.0 & 0.61 \\
\hline $\mathrm{N}=48$ & 31.1 & 0.64 & 26.6 & 0.69 & 35.0 & 0.59 \\
\hline $\mathrm{N}=60$ & 32.8 & 0.64 & 27.1 & 0.7 & 35.8 & 0.59 \\
\hline $\mathrm{N}=72$ & 34.1 & 0.58 & 28.3 & 0.67 & 36.6 & 0.58 \\
\hline
\end{tabular}

As shown in the chart, the cut size and classification accuracy had some fluctuations as the number of blades increased, indicating the existence of a better number of blades. When $\mathrm{N}<36$, the Tromp curve was flat, the classification accuracy was low, and the classifying effect of the classifier on material particles was poor. This was mainly due to the existence of an inertia anti-vortex between the blades, resulting in the phenomenon of back-mixing in the side of the receding surface. Hence, the material could not be classified in time. Meanwhile, due to the large blade spacing, the radial velocity in the channel was small. As the particle size increased, the effect of centrifugal force on the particles increased. Hence, the probability for material particles to enter the rotor cage and complete the classification decreased. When $\mathrm{N}>36$, with the increase in the number of blades, the classification efficiency of materials with a smaller particle size improved, but the classification efficiency of materials with a larger particle size rapidly decreased. Moreover, the greater the number of blades, the faster the decrease rate. Therefore, the classification accuracy decreased with the increase in the number of blades at different working conditions. This was mainly 
because as the number of blades increased, the blade spacing decreased, the radial velocity between blades increased, and the inertial anti-vortex strength became weakened. The fine particles were classified according to the better airflow carrying properties. Hence, the classification efficiency for the fine particles improved. However, a narrow rotor cage channel could lead to an increased chance of collision between materials and blades, which would increase the randomness of the material classification and decrease the classification efficiency of materials with a larger particle size. When $\mathrm{N}=36$, no polarization of the Tromp curve was observed, and a smaller cut size was obtained at higher classification accuracy, which was better for improving the classification performance and optimizing the particle grade.

\subsection{Effect of the Blade Profile on the Flow Field Distribution between Blades}

The movement of airflow in a rotor cage channel presents a typical forced vortex motion. On one hand, the airflow is driven by the rotor cage blade to do rotational movement; on the other hand, this moves inward under the action of the central negative pressure [30]. In this case, the classification of material particles mainly relies on the air drag force and centrifugal force, and both of these forces come from the airflow. Therefore, achieving a high classification accuracy is necessary to ensure that the flow field between blades is uniform and stable. By analyzing the flow characteristics of the airflow in the rotor cage channel, the influence of the blade profile on the flow field distribution was investigated, and a blade profile suitable for the high-efficiency rotor classifier was designed.

Due to the existence of a vortex at the bottom of the rotor cage, two cross-sections at the axial positions of $Z=-37.5$ and -75 of the rotor cage were chosen as the object of study. Figure 10 presents the cloud diagram for the radial velocity distribution and relative velocity streamline of airflow corresponding to the straight blade profile. The negative values of velocity in the figure indicate that the radial velocity direction pointed toward the center of the rotor cage, while the positive values of velocity indicate that the radial velocity direction pointed outward. As shown in Figure 10a, the flow field in the channel was seriously polarized, and a large velocity gradient was present and could reach up to $-6-0 \mathrm{~m} / \mathrm{s}$. Hence, the coarse particles could easily enter the rotor cage through the side of the proceeding surface, and these were collected as fine powder. The fine particles were easily carried by the airflow to deflect and collide with the blades, making it difficult to pass through the rotor cage. This was mainly due to the airflow in the channel induced by the rotor cage blade forced action, which formed a negative pressure area in the receding surface. This made it easy to form an inertia anti-vortex between the blades. As shown in Figure 10b, the relative velocity of the airflow on the proceeding side was greater than that on the receding side at different axial positions of the rotor cage, and a partial inertial antivortex was formed on the side of the receding surface. Affected by the inertial anti-vortex and deflection, the airflow impacted the blade at a larger angle. This increased the chance for the material particles to hit the blades, which was not conducive for classification.

By analyzing the flow characteristics of the airflow in the rotor cage channel, the blade profile of the streamlined blade was determined. The movement of the airflow in the rotor cage channel exhibited a composite movement that could be decomposed into implicate and relative motion. The implicated velocity is represented by $\vec{u}$, the relative velocity is represented by $\vec{w}$, and the absolute velocity is represented by $\vec{v}$. According to these three velocity vectors, the velocity triangle could be drawn at the blade inlet and outlet, as shown in Figure 11. In the figure, $\alpha_{\text {in }}$ refers to the inlet installation angle, i.e., the angle between the tangent line at the airflow inlet and the tangent line of the blade; $\beta_{1}$ refers to the relative velocity angle, which is the angle between the relative velocity vector and the tangent line at the airflow inlet; and $i_{1}$ refers to the impact angle, which is the angle between the tangent line of the blade and the relative velocity vector. 

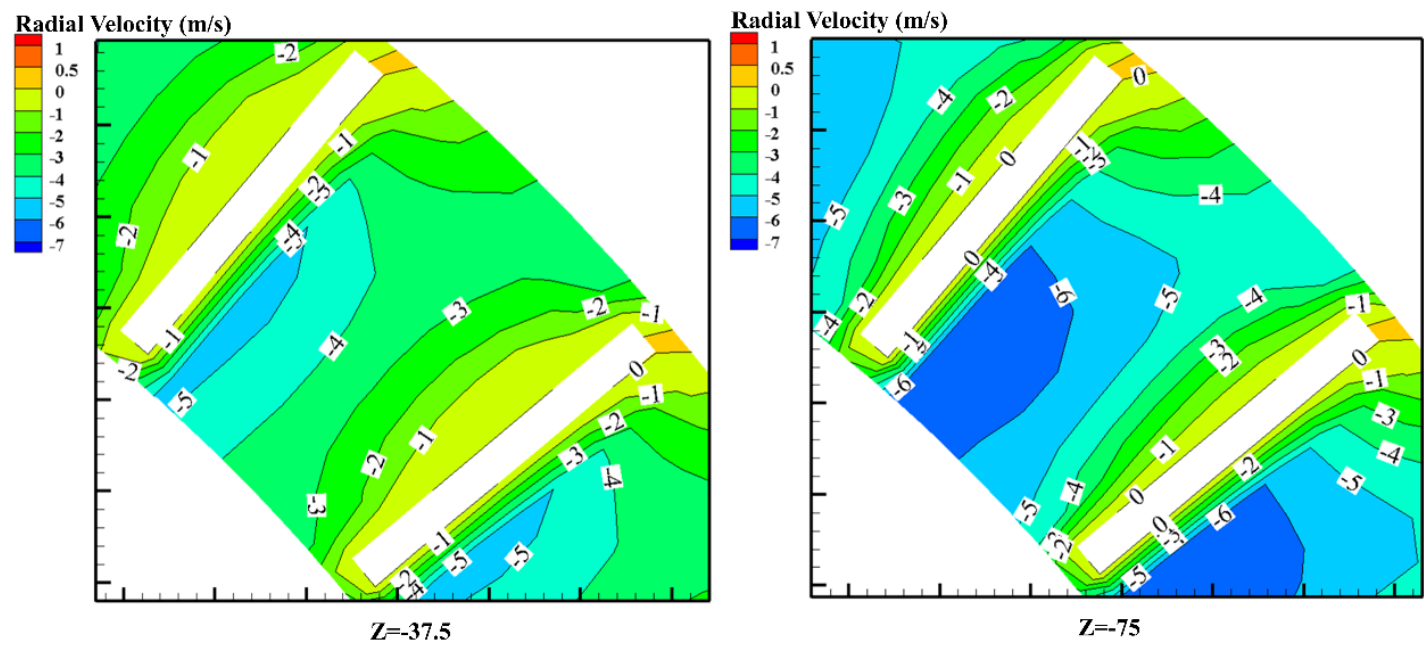

(a) Radial velocity cloud diagram
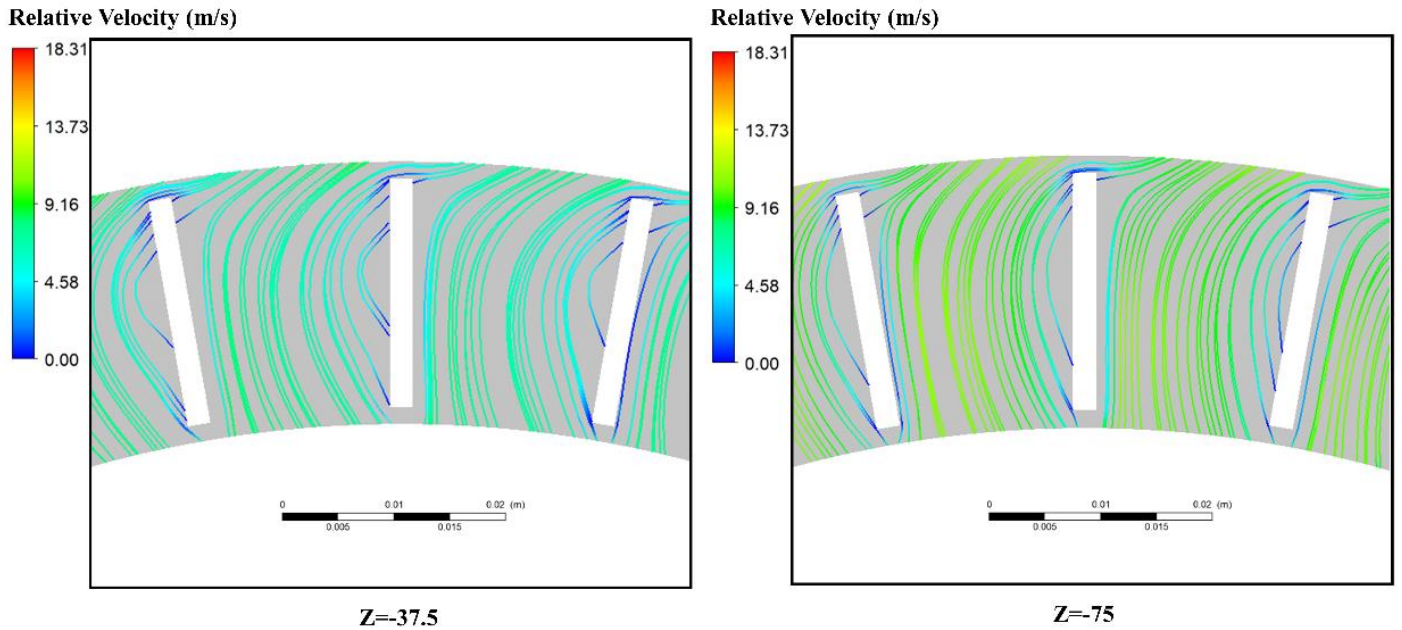

(b) Relative velocity streamline diagram

Figure 10. The cloud diagram for the radial velocity distribution and relative velocity streamline corresponding to the straight blade.

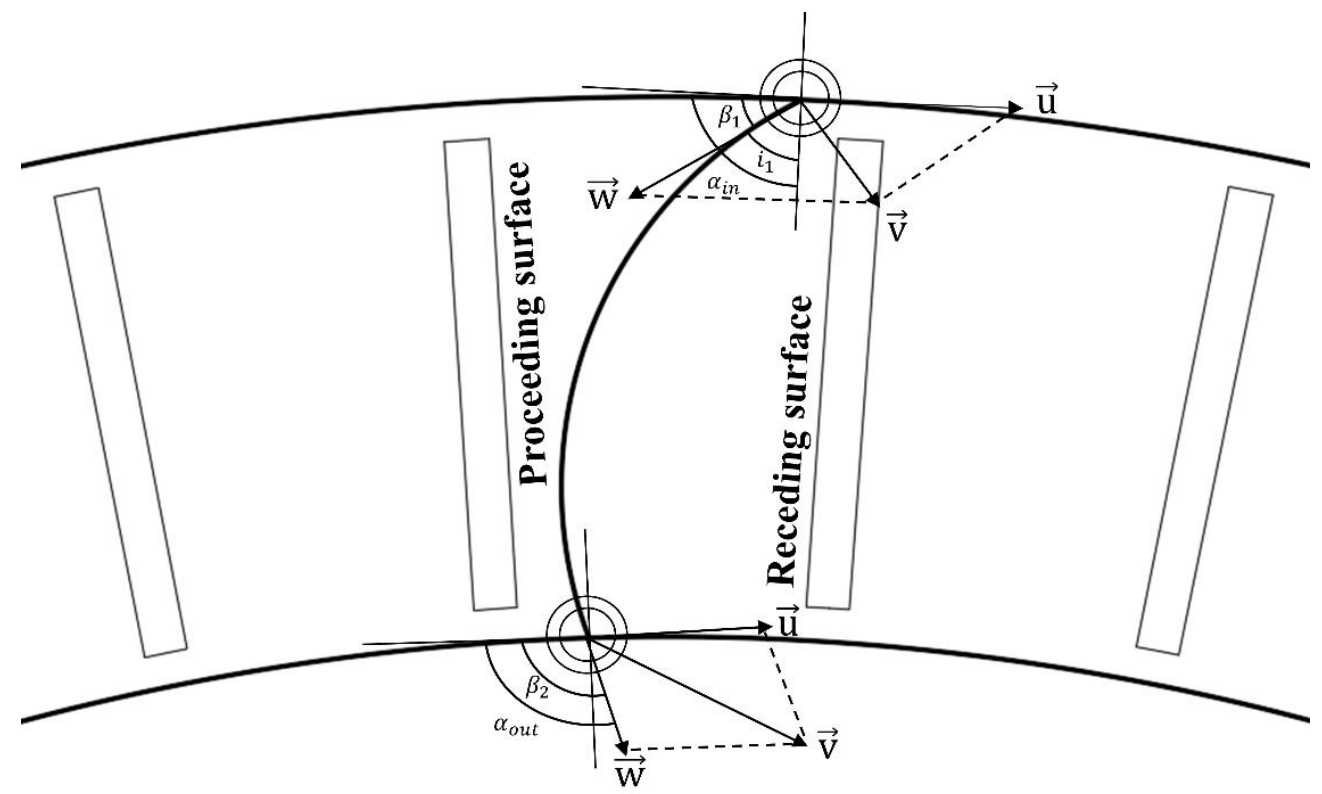

Figure 11. Analysis of the flow characteristics of the airflow in the rotor cage channel. 
Since a conventional rotor cage has a straight blade profile, the inlet installation angle $\alpha_{\text {in }}$ and outlet installation angle $\alpha_{\text {out }}$ were both $90^{\circ}$. The relative velocity angle was calculated using Fluent 19.0, in which the relative velocity angle $\beta_{1}$ at the inlet and $\beta_{2}$ at the outlet were $38^{\circ}$ and $100^{\circ}$, respectively. Thus, the airflow impact angle $i_{1}$ at the inlet and $i_{2}$ at the outlet were determined to be $52^{\circ}$ and $-10^{\circ}$, respectively. According to the energy equation, it is clear that the greater the impact angle of airflow, the greater the resistance and energy loss. Therefore, the inlet angle of the streamlined blade was set to $38^{\circ}$ in order to reduce the impact of airflow on the blade. In addition, the outlet installation angle was set to $90^{\circ}$, because the airflow in the blade end of the impact angle was small and the impact on the blade could be negligible. Meanwhile, the deflection of the blade profile could be reduced to facilitate the processing and manufacturing. According to the blade inlet and outlet installation angle, the streamlined blade was drawn using the single arc method, as shown in Figure 12.

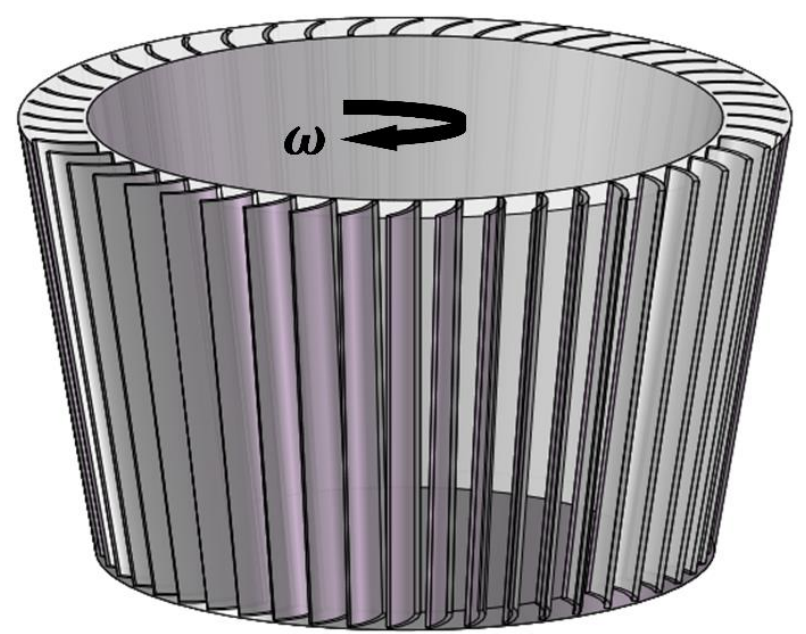

Figure 12. Schematic diagram for the streamlined blade.

The cloud diagram for the radial velocity distribution and relative velocity streamlines corresponding to the streamlined blade is shown in Figure 13. In Figure 13a, it can be observed that the velocity gradient in the flow channel was significantly reduced and that the radial velocity basically stabilized from -5 to $-1 \mathrm{~m} / \mathrm{s}$. Furthermore, the flow field distribution was more uniform and the inertial anti-vortex disappeared. In Figure 13b, it can be observed that the streamlines in the different axial positions of the rotor cage were basically coincident with the blade profile. The airflow impact angle at the inlet was basically $0^{\circ}$, which reduced the impact of the airflow on the blade. The airflow at the outlet was also in good agreement with the blades, and there were no large deflection occurrences. The improvement of the inner flow field was mainly because the streamlined blade profile was more compatible with the flow characteristics of the airflow, reducing the aerodynamic resistance. This would be beneficial for the conveyance and classification of material particles, reducing the chance of particle back-mixing.

According to the discrete phase simulation results, $\mathrm{d}_{50}$ and $\mathrm{K}$ are illustrated in Table 5 . For each operating condition, the simulation results indicated that the streamlined blade rotor cage reduced the cut size by $4.7-6.3 \%$, while the classification accuracy remained basically unchanged. The cut size reduction was mainly due to the flow field in the rotor cage channel, which became more uniform, and the velocity gradient decreased. As a result, the drag force of the particles decreased and materials with a larger particle size had difficulties in passing through the rotor cage. However, a range of application exists for the inlet installation angle at simulated operating conditions. When the inlet air velocity and rotational speed greatly differed from the simulated working condition, the inlet installation angle and airflow incidence angle were no longer equal, which was not 
conducive for classification. Therefore, the application range of the streamlined blade rotor cage needs to be further studied.
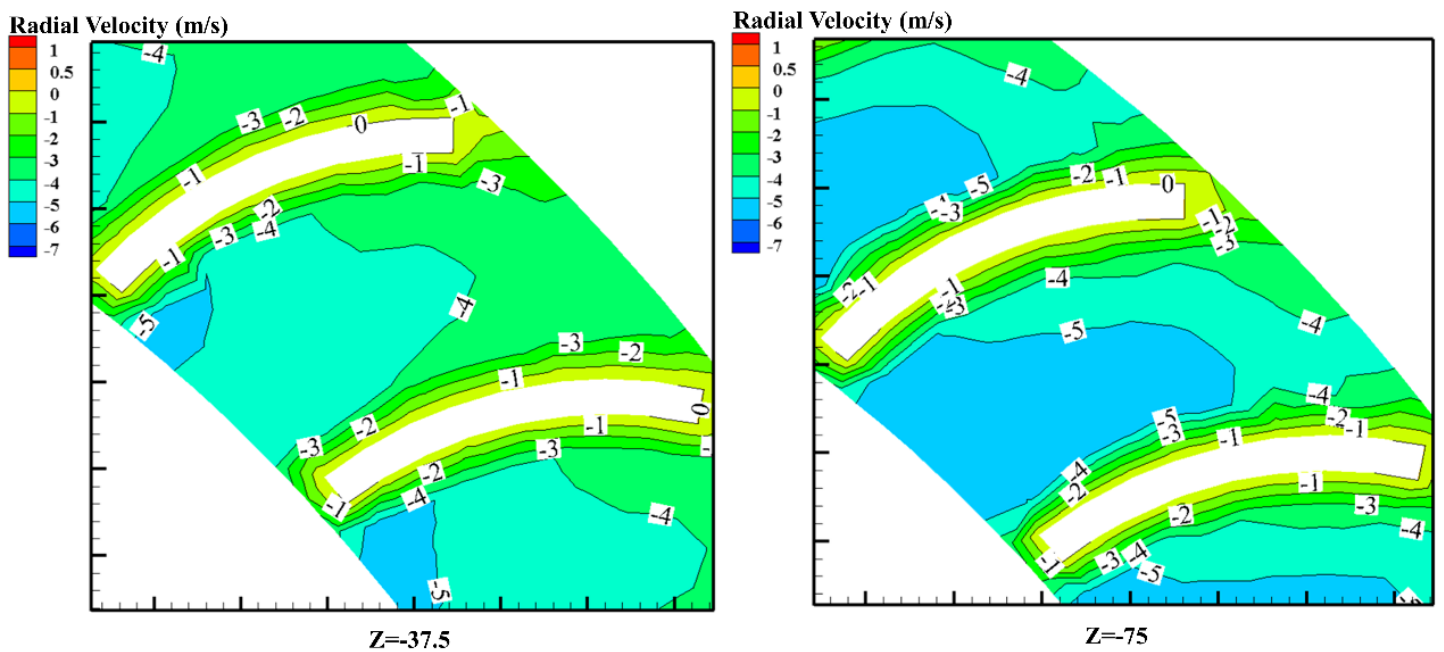

(a) Radial velocity cloud diagram
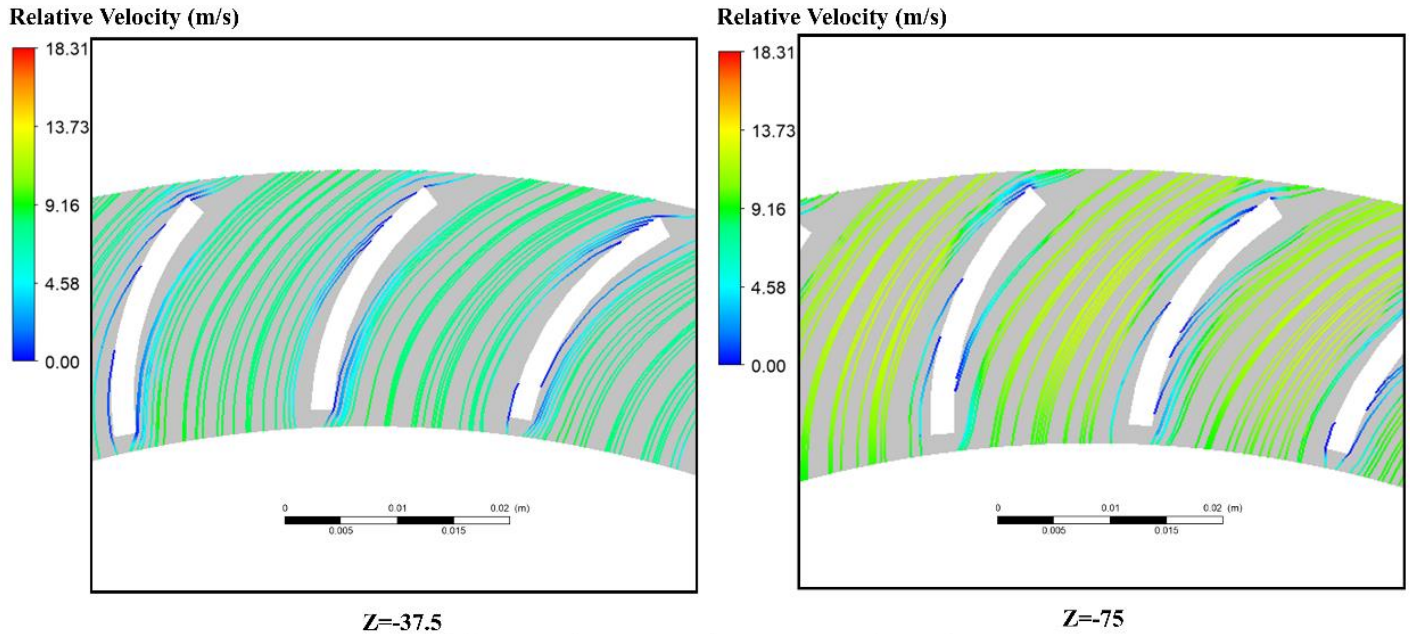

(b) Relative velocity streamline diagram

Figure 13. The cloud diagram for the radial velocity distribution and relative velocity streamline corresponding to the streamlined blade.

Table 5. The cut size and classification accuracy for the different blade profiles.

\begin{tabular}{cccccc}
\hline \multirow{2}{*}{$\mathbf{v}(\mathbf{m} / \mathbf{s})$} & $\mathbf{n}(\mathbf{r p m})$ & \multicolumn{2}{c}{ Cut Size $\left(\mathbf{d}_{\mathbf{5 0}}\right)$} & \multicolumn{2}{c}{ Classification Accuracy $(\mathbf{K})$} \\
\cline { 3 - 6 } & & $\begin{array}{c}\text { Straight } \\
\text { Blade }\end{array}$ & $\begin{array}{c}\text { Streamlined } \\
\text { Blade }\end{array}$ & $\begin{array}{c}\text { Straight } \\
\text { Blade }\end{array}$ & $\begin{array}{c}\text { Streamlined } \\
\text { Blade }\end{array}$ \\
\hline 13.8 & 500 & 29.7 & 28.3 & 0.68 & 0.68 \\
13.8 & 600 & 25.5 & 24.1 & 0.73 & 0.72 \\
16.2 & 500 & 35.0 & 32.8 & 0.61 & 0.61 \\
\hline
\end{tabular}

\subsection{Comparison of the Simulation and Experiment Results}

In order to verify the reliability of the simulation results, powder classification experiments were conducted and the simulation results were compared with the experimental data. An optimized rotor cage (a conical rotor cage with 36 straight blades) was selected for the classification experiments. The experiment and simulation were performed at different inlet velocities and rotary speeds, including 13.8-500, 13.8-600, and 16.2-500. The particle size distribution when using calcium carbonate as the raw material is presented in 
Figure 14. The blue line represents the cumulative distribution curve, while the red line represents the distribution frequency of the particles. After each test, the coarse and fine powders were weighed for mass balance. The samples were analyzed using a laser particle sizer, and then the partial classification efficiency of the high-efficiency rotor classifier was calculated.

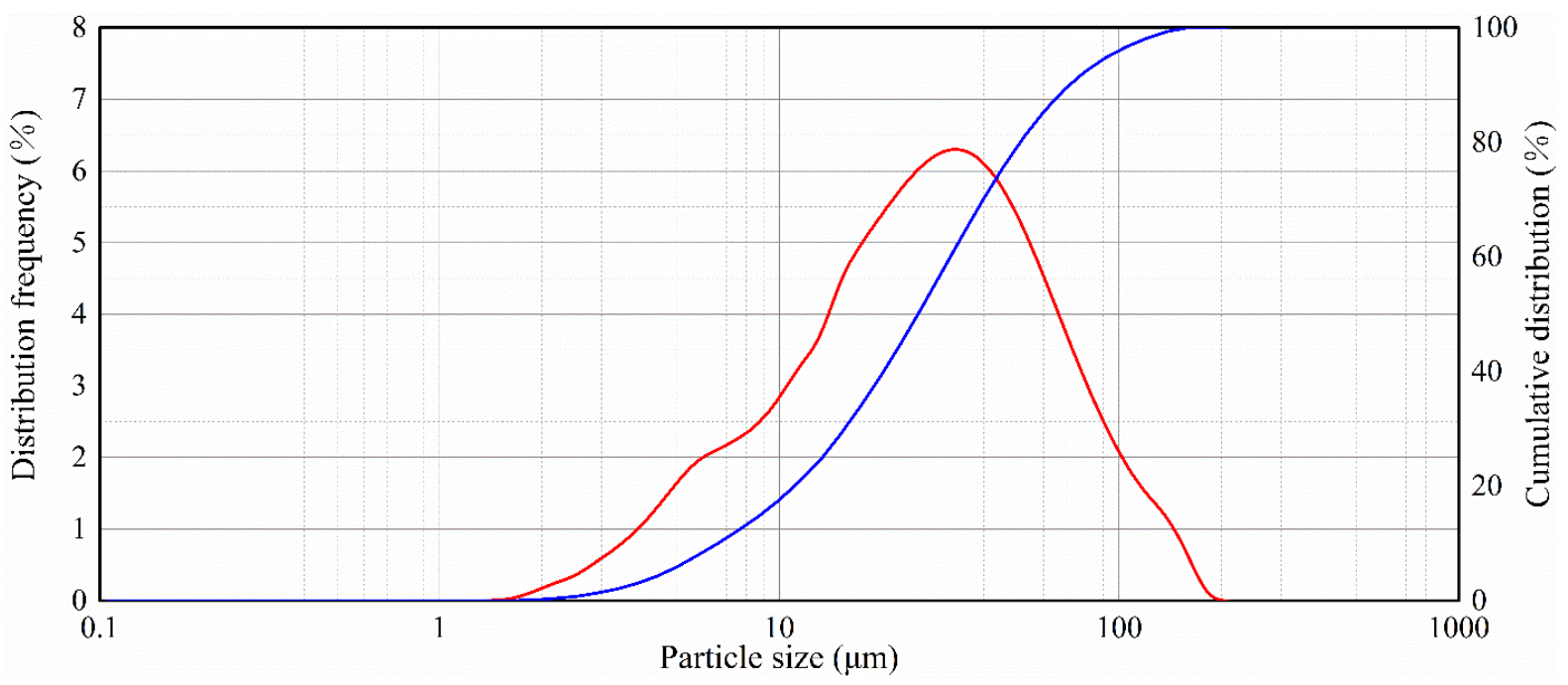

Figure 14. Particle size analysis report for the calcium carbonate raw material.

The partial classification efficiency (Tromp curve) for the simulation and experiment is presented in Figure 15. A certain difference between the simulated and experimental Tromp curves can be observed in the figure, mainly in the fishhook effect, but the overall trend was the same. The experimentally obtained Tromp curves exhibited the same S-shaped trend, presenting a fishhook effect. As the particle size increased, the partial classification efficiency curve presented a trend of initially decreasing and subsequently increasing, while the fishhook dip appeared at approximately $6 \mu \mathrm{m}$. This phenomenon could not be predicted by the DPM model, which was due to the neglect of particle interactions during the simulation, resulting in a higher classification accuracy for the simulation compared to that of the experiment. A comparison between the simulated results and the experimental data is presented in Table 6. It can be observed that the cut size and classification accuracy obtained from the simulation were in better agreement with the experiment, with maximum errors of $9.4 \%$ and $9.8 \%$, respectively. In addition, the variation of the pressure drop was well-predicted, with a maximum error of $6.0 \%$. Since the relative errors were all within $10 \%$, the computational model was correct and reasonable for predicting the performance of the classifier. However, the effect of the streamlined blades on the classification performance of the high-efficiency rotor classifier needs to be further verified. The application range of streamlined blades, as well as the effect on the fishhook effect and classification accuracy, are worthy of further studies.

Table 6. The cut size, classification accuracy, and pressure drop for the experiment and simulation.

\begin{tabular}{ccccc}
\hline Parameter & $\mathbf{v}(\mathbf{m} / \mathbf{s})-\mathbf{n}(\mathbf{r p m})$ & Simulation Value & Experimental Value & Relative Error $(\%)$ \\
\hline \multirow{2}{*}{ Cut size $\left(\mathrm{d}_{50}\right)$} & $13.8-500$ & 29.7 & 32.5 & 9.4 \\
& $13.8-600$ & 25.5 & 27.0 & 5.9 \\
Classification accuracy $(\mathrm{K})$ & $16.2-500$ & 35.0 & 36.6 & 4.6 \\
& $13.8-500$ & 0.68 & 0.62 & 8.8 \\
Pressure drop $(\Delta \mathrm{P})$ & $13.8-600$ & 0.73 & 0.66 & 9.6 \\
& $16.2-500$ & 235.5 & 249.3 & 5.9 \\
& $13.8-500$ & 260.3 & 273.6 & 5.1 \\
\end{tabular}




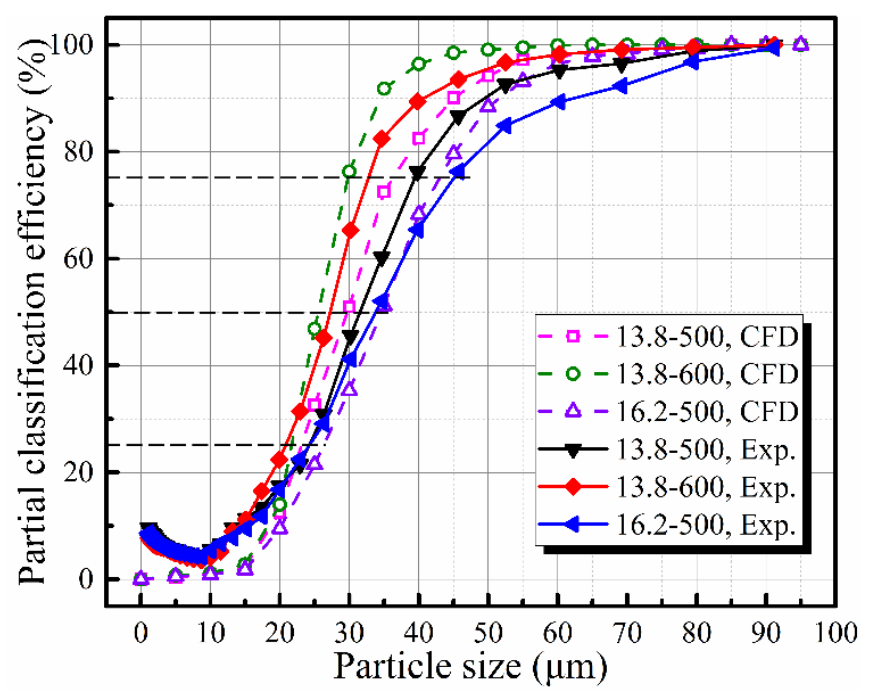

Figure 15. Partial classification efficiency curves for the experiment and simulation.

\section{Conclusions}

In the present study, a high-efficiency rotor classifier was designed, and the flow behavior in the classifier was numerically simulated by the ANSYS-Fluent 19.0 software. The effects of rotor cage shape, the number of blades, and the blade profile on the inner flow field distribution and the classification performance of the classifier were investigated. The conclusions are as follows:

(1) The high-efficiency rotor classifier employed wind feed, which reduced the content of coarse particles and increased the dispersion of materials. This effectively solved the problems of uneven materials spreading and high dust concentration. Meanwhile, since no guide vanes were equipped in the high-efficiency rotor classifier, there was no need to consider the wear of the guide vanes.

(2) According to the analysis of the flow field characteristics, it could be seen that the vortices existed at the bottom of the rotor cage and the column rotor cage was not conducive to maintaining the uniformity of airflow distribution near the classification surface. On the contrary, the conical rotor cage not only reduced the vortex at the bottom of the rotor cage but also improved the uniformity of the flow field.

(3) As the number of blades increased, the cut size and classification accuracy exhibited some fluctuations, indicating the existence of a better number of blades. A reasonable number of blades resulted in a more uniform flow field distribution between the blades and no polarization of the Tromp curve. This contributed to the improvement of classification performance and the optimization of particle gradation.

(4) Compared with a traditional straight blade, a streamlined blade was found to eliminate the inertial anti-vortex between blades, reducing the velocity gradient and making the flow field distribution more uniform. Meanwhile, the streamline of airflow in the channel basically coincided with the blade profile, which reduced the impact of the airflow on the blades. This is beneficial for the conveyance and classification of materials.

(5) The experimental results indicated that the DPM could well-predict the $\mathrm{d}_{50}$ and K values of the high-efficiency rotor classifier. However, the experimental partial classification efficiency presented an S-shape, known as 'fishhook,' which is a phenomenon that cannot be predicted. 


\section{Nomenclature/Abbreviations}

\begin{tabular}{ll}
\hline $\mathrm{d}_{25}$ & Particle diameter at $25 \%$ penetration $(\mu \mathrm{m})$ \\
$\mathrm{d}_{50}$ & Particle diameter at $50 \%$ penetration, cut size $(\mu \mathrm{m})$ \\
$\mathrm{d}_{75}$ & Particle diameter at $75 \%$ penetration $(\mu \mathrm{m})$ \\
$\Delta \mathrm{P}$ & Pressure drop $(\mathrm{Pa})$ \\
$\mathrm{K}$ & Classification accuracy $(-)$ \\
$\mathrm{v}$ & Inlet air velocity $(\mathrm{m} / \mathrm{s})$ \\
$\mathrm{n}$ & Rotor cage speed $(\mathrm{rpm})$ \\
$\mathrm{N}$ & The number of blades $(-)$ \\
$\vec{u}$ & Implicated velocity $(\mathrm{m} / \mathrm{s})$ \\
$\vec{v}$ & Absolute velocity $(\mathrm{m} / \mathrm{s})$ \\
$\vec{w}$ & Relative velocity $(\mathrm{m} / \mathrm{s})$ \\
$\alpha_{i n}$ & Inlet installation angle $\left(^{\circ}\right)$ \\
$\beta_{1}$ & Inlet relative velocity angle $\left(^{\circ}\right)$ \\
$i_{1}$ & Inlet impact angle $\left(^{\circ}\right)$ \\
$\alpha_{\text {out }}$ & Outlet installation angle $\left(^{\circ}\right)$ \\
$\beta_{2}$ & Outlet relative angle $\left(^{\circ}\right)$ \\
$i_{2}$ & Outlet impact angle $\left(^{\circ}\right)$ \\
$\mathrm{CFD}$ & Computational fluid dynamics \\
$\mathrm{DPM}$ & Discrete phase model \\
$\mathrm{MRF}$ & Multiple reference coordinates system model \\
$\mathrm{DRW}$ & Discrete random walk model \\
$\mathrm{RSM}$ & Reynolds stress model \\
\hline
\end{tabular}

Author Contributions: X.M. was responsible for numerical simulations and analysis of experimental results, writing and editing the manuscript. F.J. was responsible for modeling the computational model and model validation. Y.F. supervised the research and provided initial research ideas. C.C. supervised and directed the research. All authors have read and agreed to the released version of the manuscript.

Funding: This work was supported by the general institute of building materials research of China (No.2017YFC0210801), and the Priority Academic Program Development of Jiangsu Higher Education Institutions (PAPD).

Conflicts of Interest: The authors declared no potential conflict of interest with respect to the research, authorship, and/or publication of this article.

\section{References}

1. Eswaraiah, C.; Angadi, S.I.; Mishra, B.K. Mechanism of particle separation and analysis of fish-hook phenomenon in a circulating air classifier. Powder Technol. 2011, 218, 57-63. [CrossRef]

2. Guizani, R.; Mhiri, H.; Bournot, P. Effects of the geometry of fine powder outlet on pressure drop and separation performances for dynamic separators. Powder Technol. 2016, 314, 599-607. [CrossRef]

3. Barimani, M.; Green, S.; Rogak, S. Particulate concentration distribution in centrifugal air classifiers. Miner. Eng. 2018, 126, 44-51. [CrossRef]

4. Shapiro, M.; Galperin, V. Air classification of solid particles: A review. Chem. Eng. Process. Process Intensif. 2004, 44, 279-285. [CrossRef]

5. Lu, D.; Fan, Y.; Lu, C. Advances in research on granular air classification. China Powder Sci. Technol. 2020, 26, 11-24.

6. Lu, J.; Xia, J.; He, T. Air flow field characteristics analyzing and classification process of the turbo classifier. J. Chin. Ceram. Soc. 2003, 31, 485-489.

7. Yang, Q.L.; Liu, J.X.; Zhou, Y.B. Flow Field Characteristic Analyses of a Turbo Air Classifier's Rotor Cage and its Structurally Improved Counterpart. Adv. Mater. Res. 2009, 782, 59-67. [CrossRef]

8. Xing, W.; Wang, Y.; Zhang, Y.; Yamane, Y.; Saga, M.; Lu, J.; Zhang, H.; Jin, Y. Experimental study on velocity field between two adjacent blades and gas-solid separation of a turbo air classifier. Powder Technol. 2015, 286, 240-245. [CrossRef]

9. Feng, L.; Wu, Y.; Zhang, H.; Zhang, Y.; Yue, G. Effect of air flow rate and rotational speed on vortices between neighboring blades in turbo air classifiers. J. Tsinghua Univ. Sci. Technol. 2020, 60, 493-499.

10. Huang, Q.; Liu, J.; Yu, Y. Turbo air classifier guide vane improvement and inner flow field numerical simulation. Powder Technol. 2012, 226, 10-15. [CrossRef]

11. Liu, R.; Liu, J.; Yu, Y. Effects of axial inclined guide vanes on a turbo air classifier. Powder Technol. 2015, 280. [CrossRef]

12. Yu, Y.; Ren, W.; Liu, J. A new volute design method for the turbo air classifier. Powder Technol. 2019, 348, 65-69. [CrossRef] 
13. Huang, Q.; Yu, Y.; Liu, J. Improvement on rotor cage structure of turbo air classifier and numerical simulation of inner flow field. CIESC J. 2011, 62, 1264-1268.

14. He, F.; Fang, Y.; Qin, J.; Chai, S. Simulation and Study on Flow Field Characteristics of Annular Regions in New Tpye Turbo Classifier. Bull. Chin. Ceram. Soc. 2016, 35, 4128-4132.

15. Zeng, Y.; Zhang, S.; Zhou, Y.; Li, M. Numerical Simulation of a Flow Field in a Turbo Air Classifier and Optimization of the Process Parameters. Processes 2020, 8, 237. [CrossRef]

16. Yu, Y.; Kong, X.; Liu, J. Effect of rotor cage's outer and inner radii on the inner flow field of the turbo air classifier. Materialwiss Werkst. 2020, 51, 908-919. [CrossRef]

17. Jiang, D.; Han, J.; Gao, Y. Influence of distance between cage blades on flow field in cylindrical cage powder classifier. Min. Process. Equip. 2013, 41, 91-95.

18. Chang, L.; Zuobing, C.; Weili, Z.; Chenggang, Y.; Ya, M.; Yongjie, Y.; Qiang, X.; Babak, S. Effects of Blade Parameters on the Flow Field and Classification Performance of the Vertical Roller Mill via Numerical Investigations. Math. Probl. Eng. 2020, 2020. [CrossRef]

19. Spoetter, C.; Legenhausen, K.; Weber, A.P. Influence of the Paddle Geometry on the Fluid Flow and Particle Movement of a Deflector Wheel Separator. Chem. Ing. Tech. 2018, 90, 493-506.

20. Jiang, D.; Tao, R. Influence of Structure of Cage Blades on Airflow Characteristic in O-sepa Classifier. Mech. Eng. Autom. 2014, $187,22-23$.

21. Xu, N.; Li, G.; Huang, Z. Numerical simulation of particle motion in turbo classifier. Particuology 2005, 3, 275-278. [CrossRef]

22. Ren, W.; Liu, J.; Yu, Y. Design of a rotor cage with non-radial arc blades for turbo air classifiers. Powder Technol. 2016, $292,46-53$. [CrossRef]

23. Ren, W.; Liu, J.; Yu, Y. Design of Rotor Cage with Arc-blade for the Turbo Air Classifier. J. Mech. Eng. 2016, 52, 195-201. [CrossRef]

24. Zhao, H.; Liu, J.; Yu, Y. Effects of the impeller blade geometry on the performance of a turbo pneumatic separator. Chem. Eng. Commun. 2018, 205, 1641-1652. [CrossRef]

25. Dou, H.; Qin, Z.; Wang, W.; Chai, X.; Yu, T.; Zhao, J. Research and Application of a U Shape Blades Classifier. Cem. Technol. 2018, 204, 34-39.

26. Wu, S.; Liu, J.; Yu, Y. Design of a new double layer spreading plate for a turbo air classifier. Powder Technol. 2017, 312, 277-286. [CrossRef]

27. Sun, Z.; Sun, G.; Liu, J.; Yang, X. CFD simulation and optimization of the flow field in horizontal turbo air classifiers. Adv. Powder Technol. 2017, 28, 1474-1485. [CrossRef]

28. Zhanpeng, S.; Longlong, L.; Chunyu, L.; Guang, Y. Structural optimization of vortex finder for a centrifugal air classifier. Chem. Eng. Res. Des. 2021, 166, 220-226.

29. Galletti, C.; Rum, A.; Turchi, V.; Nicolella, C. Numerical analysis of flow field and particle motion in a dynamic cyclonic selector. Adv. Powder Technol. 2020, 31, 1264-1273. [CrossRef]

30. Scheit, C.; Karic, B.; Becker, S. Effect of blade wrap angle on efficiency and noise of small radial fan impellers-A computational and experimental study. J. Sound Vib. 2011, 331, 996-1010. [CrossRef] 